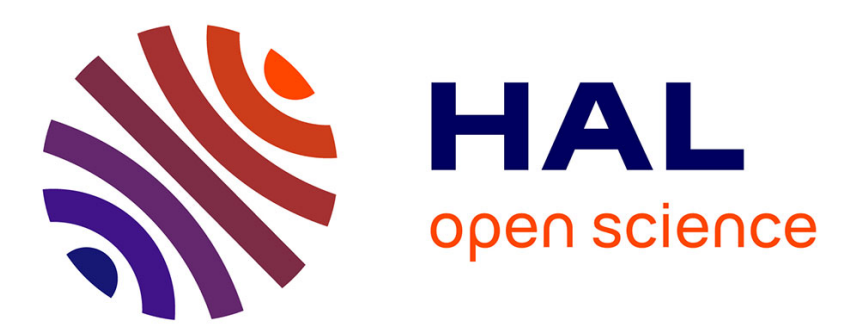

\title{
Synthèse sur la pratique des dépôts métalliques au Bronze moyen en Médoc (Gironde, France) à la lumière de l'analyse spatiale \\ Florian Couderc
}

\section{- To cite this version:}

Florian Couderc. Synthèse sur la pratique des dépôts métalliques au Bronze moyen en Médoc (Gironde, France) à la lumière de l'analyse spatiale. Bulletin de la Société préhistorique française, 2017, 114 (3), pp.529-552. 10.3406/bspf.2017.14805 . hal-02095357

\section{HAL Id: hal-02095357 \\ https://hal.science/hal-02095357}

Submitted on 4 Aug 2020

HAL is a multi-disciplinary open access archive for the deposit and dissemination of scientific research documents, whether they are published or not. The documents may come from teaching and research institutions in France or abroad, or from public or private research centers.
L'archive ouverte pluridisciplinaire HAL, est destinée au dépôt et à la diffusion de documents scientifiques de niveau recherche, publiés ou non, émanant des établissements d'enseignement et de recherche français ou étrangers, des laboratoires publics ou privés. 


\title{
Synthèse sur la pratique des dépôts métalliques au Bronze moyen en Médoc (Gironde, France) à la lumière de l'analyse spatiale
}

\author{
Florian COUderC
}

Résumé : La concentration exceptionnelle de dépôts métalliques du Bronze moyen 2 en Médoc est connue depuis le $\mathrm{XIX}^{\mathrm{e}}$ siècle. Cette pratique n'est pas limitée au domaine atlantique, mais la concentration de cinquante-sept dépôts sur un territoire aussi restreint en fait un cas particulier à l'échelle de la France. Le Médoc regroupe à ce titre plus de la moitié des dépôts découverts en Aquitaine. Ces dépôts se caractérisent par des haches en bronze massives, principalement du type médocain, mais aussi de productions exogènes, telles que les haches à talon de types breton, normand et beaucoup plus rarement du Centre-Ouest. Au total ce sont plus de $660 \mathrm{~kg}$ de bronze qui ont été découverts en Médoc depuis le XIX ${ }^{\mathrm{e}}$ siècle. Pourtant, cette région ne dispose d'aucun gisement métallifère. Les échanges avec l'ensemble du domaine atlantique sont bien attestés par les productions métalliques venues de ces régions et qui se retrouvent dans les dépôts du Médoc. La relative rareté des productions continentales, telles que les haches du type du Centre-Ouest ou d'Orléans, prouve que les sociétés médocaines étaient prioritairement tournées vers l'Océan.

La situation géographique, entre l'Océan et le fleuve Garonne, a contribué au développement des échanges à longue distance. La place stratégique qu'occupait le Médoc au Bronze moyen a donc permis aux populations de la péninsule de s'approvisionner en ressources métalliques. La question se pose alors de savoir quelles contreparties ces sociétés pouvaient proposer dans les échanges. Des indices de production de sel sont attestés plus au sud à la dune du Pilat pour le Bronze moyen, mais également sur le littoral médocain pour des périodes plus récentes. Il s'agit d'une des possibilités, mais les données sur l'habitat et l'artisanat manquent cruellement pour pousser la réflexion. La quasi disparation de la pratique des dépôts au Bronze final coïncide avec une modification de l'environnement. La structure économique a pu à cette occasion être ébranlée, ne permettant plus aux sociétés de l'âge du Bronze du Médoc de disposer des ressources nécessaires au maintien des échanges à longue distance et à leur approvisionnement en ressource métallique.

L'évolution de l'environnement autour de l'estuaire de la Gironde est en effet très importante durant l'âge du Bronze, avec des modifications importantes du fonctionnement des marais médocains. Nous pouvons mettre en évidence que la pratique des dépôts métalliques au Bronze moyen se concentre exclusivement autour des marais tidaux, dont nous savons grâce aux études paléoenvironnementales qu'ils étaient en eaux à cette époque. Ce lien fort entre les dépôts et les zones humides questionne sur la relation qu'ils pouvaient entretenir. Nous pouvons envisager un rôle symbolique, en lien avec les eaux divinisées, qui est attesté par ailleurs notamment au Bronze final et au premier âge du Fer, ou bien encore à une structuration pragmatique du territoire. Il semble qu'il y ait une récurrence de cette pratique sur des mêmes espaces, comme en témoignent certaines découvertes réalisées autour de Pauillac, ou encore de Soulac-sur-Mer, avec plusieurs dépôts enfouis à quelques mètres les uns des autres. Ce comportement répétitif et codifié connote une certaine forme de ritualisation des dépôts métalliques. Si l'essence même de cette pratique est difficilement compréhensible pour notre mode de pensée contemporain, le cas du Médoc permet d'entrevoir des pistes de réflexion qui devront être confrontée à l'avenir aux autres régions de la façade atlantique.

Mots-clefs : Aquitaine, Médoc, Bronze moyen, dépôt métallique, haches, échanges, paléoenvironnement.

\begin{abstract}
We have known since the 19th century that the Médoc region offers an exceptional number of metal hoards dating from the Middle Bronze Age 2. Metal hoarding is not restricted to the Atlantic coastal area but a concentration of fifty-seven hoards - more than half of the Aquitaine hoards - in such a limited area clearly makes it exceptional. Many isolated objects have also been found in the Médoc region, but we do not know why they were deposited there. Were they a discreet practice of metal hoarding, or just lost objects? These Médoc hoards are made up of massive bronze axes, mainly of the Médoc type, but palstave axes from Brittany and Normandy, and more rarely from the Centre-Ouest region, can also be found. In total, over 660 kilos of bronze artefacts have been discovered in the Médoc region since the 19th century, even though the area had no copper or tin deposits. This is further proof of the trade that existed along the Atlantic coastline. The relative scarcity of productions from the Centre-West or from Orléans indicates that the Médoc societies were turned towards the ocean, and the location, between the ocean and the River Garonne, contributed
\end{abstract}


to the development of long-distance exchanges. This strategic position, with the difficulty of navigating southwards in the Bay of Biscay and the proximity with the River Garonne, allowed the populations in the Médoc region access to metal resources, raw materials as well as finished products. Furthermore, some signs of bronze production have been discovered in Médoc, such as moulds of flanged axes and an anvil on the Lède du Gurp site (Soulac-sur-Mer). What did those populations give in exchange for such resources? Indications of salt production during the Middle Bronze Age were found south of the Dune du Pilat, as well as signs of later production along the Médoc coastline. This could answer the question, although little is known about domestic crafts and local activities.

The environmental change around the Gironde estuary during the Late Bronze Age led to important alterations to the Médoc marshlands. The disappearance of the practice of metal hoarding during the Late Bronze Age in Médoc coincides with this transformation of the environment. In fact, during the Late Bronze Age and the Iron Age, the Médoc landscape became increasingly humid. This change may have disrupted the economic structure of Middle Bronze Age societies, leading to an interruption in long-distance exchanges and consequently in the supply of metallic resources. This hypothesis may however mask the possibility of a rupture in the social structure of these societies. Metal goods were probably controlled by an elite hit by a power crisis, which led to a drop in metal supply and metal hoarding. At the same time, many hoards were deposited in other parts of the Atlantic area, such as Brittany, Normandy and the Loire Atlantique département. This difference between North and South demonstrates that practices and societies changed dramatically in the Médoc between the Middle and the Late Bronze Age.

It is possible to prove that metal hoards during the Middle Bronze Age were exclusively situated near tidal marshes which were not dry at the time, as is indicated by palaeo-environmental studies. There is a strong link between hoards and wetlands, but what was the connection between the two? We can envisage a symbolic role, linked to the sacred nature of water - a possibility also noted during the Late Bronze Age and the early Iron Age - or more simply the result of a pragmatic delimitation of territory. It would seem that this was a recurrent practice in those areas: hoards have been discovered only a few metres apart, in Pauillac and Soulac-sur-Mer. This standardised behaviour implies a certain ritualization of metal hoarding. Even if the meaning or motivation behind such practices is difficult to apprehend for our modern way of thinking, the case study of the Médoc region can pave the way for new hypotheses. It would be interesting to try this model out on other sites along the Atlantic coastal area for the Middle Bronze Age.

Keywords : Aquitaine, Médoc, Middle Bronze age, metal hoarding, axes, trade, paleoenvironmental.

$\mathrm{L}$ E MéDoc est situé dans le Sud-Ouest de la France, dans le département de la Gironde, à $10 \mathrm{~km}$ au nord-ouest de Bordeaux (fig. 1). Ce territoire est pris entre l'estuaire de la Gironde à l'est et l'océan atlantique à l'ouest. Le fleuve se jette dans l'océan au nord de la pointe du Médoc, près de la ville de Soulacsur-Mer. La péninsule médocaine mesure $80 \mathrm{~km}$ de long sur environ $50 \mathrm{~km}$ pour sa partie la plus large. Cette position entre l'océan et le fleuve a fortement influencé la géomorphologie et l'évolution de l'environnement du Médoc. Le paysage durant la Protohistoire était beaucoup plus humide qu'aujourd'hui et sa restitution permet d'entrevoir de nouvelles pistes de réflexion sur les modalités de constitution des dépôts métalliques à l'âge du Bronze. Le Médoc concentre en effet un nombre exceptionnel de dépôts de la fin du Bronze moyen 1 et surtout du Bronze moyen 2. À l'échelle de la façade atlantique, il s'agit de la seule région qui en ait livré autant de cette période sur un si petit territoire. Au total, les dépôts regroupant plus de deux objets, sont au nombre de cinquante-sept. Il s'agit uniquement de dépôts terrestres. Pour comparaison, seuls quarante-trois dépôts sont connus pour le reste de l'ancienne région Aquitaine pour l'ensemble du Bronze moyen.

Le Bronze moyen est de loin la période la plus représentée au travers du mobilier métallique en Aquitaine. Outre le nombre important des découvertes, ces dépôts sont souvent très pondéreux, car majoritairement composés de haches à rebords. La masse totale des haches découvertes en Médoc (dépôts et objets isolés) avoisine les $660 \mathrm{~kg}$. Cette masse considérable est d'autant plus impressionnante lorsque l'on constate que le Médoc ne dispose d'aucun gisement métallifère. Cet article propose une synthèse de la pratique des dépôts métalliques du Bronze moyen en Médoc par l'analyse de leur composition, mais également à travers leurs localisations et leurs liens avec le paysage ancien.

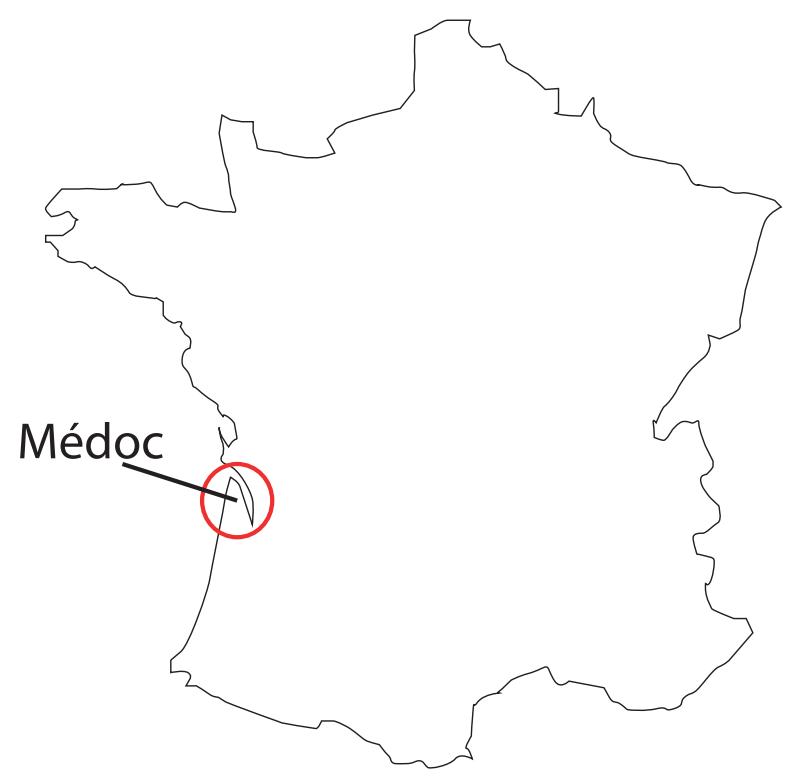

Fig. 1 - Localisation du Médoc en France.

Fig. 1 - The Médoc region in France. 


\section{HISTOIRE DE LA RECHERCHE SUR L'ÂGE DU BRONZE EN MÉDOC}

$\mathrm{D}^{\mathrm{s}}$ ès le $\mathrm{XIX}^{\mathrm{e}}$ siècle, les découvertes d'objets métalliques de l'âge du Bronze en Médoc ont intéressé les érudits locaux. De nombreux dépôts ont été trouvés et quelques notices et articles relatent ces découvertes. Nous pouvons citer les travaux du baron de Caila (17441832), ou encore ceux de François Vatar Jouannet (17651845), qui ont été les premiers à collectionner ces objets et à proposer des notices dans le Bulletin de la Société archéologique de Bordeaux (Caila 1806; Jouannet 18271828). C'est durant la deuxième moitié du XIX ${ }^{\mathrm{e}}$ siècle que François Daleau réalise une synthèse sur les « cachettes de bronziers » de Gironde (Daleau, 1876 et 1896). Il reprenait dans ses articles les différentes découvertes que lui avaient signalées des collectionneurs, tels qu'Armand Meynieu de Talais, Pouvereau de Lesparre ou encore Bonnore de Chasteignier, Dulignon-Desgranges et Noguey de Bordeaux. C'est un réel social network avant l'heure qui s'était mis en place au cours du XIX ${ }^{\mathrm{e}}$ siècle, durant lequel de nombreuses correspondances ont été entretenues et qui ont été regroupées dans un article par Hubert Sion (1989). Mais c'est véritablement avec les travaux du docteur Ernest Berchon (1825-1894) que les recherches sur l'âge du Bronze en Médoc vont prendre de l'ampleur. Il réalise une importante publication qui constitue encore aujourd'hui une base de référence pour toute recherche en Aquitaine (Berchon, 1890). Il est le premier à utiliser le terme de « médocaine » pour les haches massives à rebords découvertes en nombre dans le Médoc et que l'on retrouve dans tout le Sud-Ouest de la France. Après lui, quelques rares mentions de découvertes sont publiées par Gaston Lalanne (1862-1925) et Marcel Charrol (18741952) durant la fin du XIX ${ }^{\mathrm{e}}$ siècle et le début du $\mathrm{XX}^{\mathrm{e}}$ siècle (Lalanne, 1887; Charrol, 1912). Mais globalement, les recherches sur l'âge du Bronze en Gironde perdent de leur dynamique durant la première moitié du $\mathrm{XX}^{\mathrm{e}}$ siècle.

Ce n'est qu'à partir des années 1970 et 1980, avec les travaux d'André Coffyn, que les recherches sur l'âge du Bronze en Gironde vont reprendre de l'ampleur, avec la publication de plusieurs ouvrages et articles (Coffyn, 1972 et 1989; Coffyn et al., 1995). Par la suite, Julia RoussotLarroque va reprendre ces recherches en publiant de nombreuses découvertes inédites, et quelques rares fouilles ou sondages, dont plusieurs sur le célèbre site de la Lèdedu-Gurp à Grayan-et-l'Hôpital (Roussot-Larroque, 1988, 1989, 1997, 2001 et 2007). Plus récemment, c'est la thèse de Céline Lagarde sur les productions métalliques du Bronze moyen en Aquitaine qui fait date dans les recherches sur l'âge du Bronze moyen aquitain (Lagarde-Cardona, 2012). Ces travaux récents reprennent la quasi totalité des découvertes anciennes et récentes, certaines inédites, et sont des supports importants pour cette présente publication.

Si le nombre des publications sur l'âge du Bronze en Aquitaine semble important, aucune synthèse sur la pratique des dépôts métalliques en Médoc en tant que telle n'a encore été proposée. Depuis maintenant plusieurs décennies, nous constatons une baisse significative du nombre des découvertes signalées en Médoc, alors que dans le même temps, l'utilisation des détecteurs de métaux s'amplifie. Nous pouvons raisonnablement penser qu'une part significative des dernières découvertes nous soit totalement occultée. C'est pour cela que notre corpus se compose essentiellement de découvertes anciennes, généralement fortuites, dont la qualité parfois médiocre de la documentation doit être prise en compte dans cette étude.

\section{MÉTHODE D'ÉTUDE DES DÉPÔTS DU BRONZE MOYEN 2}

$\mathrm{U}^{\mathrm{n}}$ n ensemble d'objets métalliques est considéré comme un dépôt dès lors qu'il associe au minimum deux éléments dans un ensemble clos bien identifié. Les découvertes isolées sont composées d'un ou plusieurs objets découverts dans un contexte connu, mais dont l'association entre les différents objets n'est pas avérée. Les découvertes en contexte inconnu regroupent un à plusieurs objets dont la localisation ou l'association entre les différents éléments n'est pas documentée avec précision. Plusieurs approches ont été combinées pour proposer une synthèse de la pratique des dépôts en Médoc. Une analyse matérielle des dépôts est nécessaire pour comprendre les modalités de constitution des ensembles. L'analyse statistique permettra de dégager des tendances et d'illustrer la place spécifique du Médoc dans le complexe culturel atlantique du Bronze moyen et d'ouvrir des pistes de réflexion sur les circuits d'approvisionnement en matière première et en produits finis métalliques. L'approche matérielle sera couplée à une analyse spatiale des dépôts, à partir des donnés sur le contexte paléoenvironnemental du Médoc. Nous vérifierons si cette pratique, outre une certaine homogénéité dans la composition des dépôts semble liée au paysage et à son évolution. Pour pouvoir élargir le raisonnement, les dépôts terrestres du Bronze moyen du Centre-Ouest de la France ont été pris en compte dans l'analyse des aires de répartition des productions métalliques (Gomez de Soto, 1995). Nous pourrons ainsi essayer de replacer le Médoc dans son contexte chronoculturel, tenter de comprendre pourquoi ici plus qu'ailleurs les sociétés du Bronze moyen ont déposé une telle quantité de métal, mais également proposer un nouveau regard sur la pratique des dépôts, en lien avec le paysage.

\section{GÉOMORPHOLOGIE ET PALÉOENVIRONNEMENT DU MÉDOC}

$\mathrm{L}$ e Médoc fait partie géologiquement de la formation $\checkmark$ des sables des Landes, qui s'étend sur toute la façade atlantique du Sud-Ouest de la France. La frange orientale est formée de petits coteaux calcaires, surmontés de terrasses graveleuses composées de graviers et de galets d'origine fluviale. Sur le littoral à l'ouest, de grandes 
dunes côtières se sont formées à partir du Moyen Âge. Elles s'étendent dans tout le golfe de Gascogne, sur près de $240 \mathrm{~km}$ de long, et sont parfois hautes de plusieurs dizaines de mètres. Cet environnement a fortement évolué depuis l'âge du Bronze et le paysage que nous connaissons aujourd'hui ne ressemble aucunement à celui qu'occupaient les populations protohistoriques. Plusieurs travaux nous permettent de proposer une restitution du paysage ancien en Médoc. Une thèse en géomorphologie réalisée par Bérangère Clavé en 2001, propose une synthèse de l'évolution du paysage dans le Nord de l'Aquitaine depuis l'Holocène. Plusieurs articles ont parallèlement été publiés sur ces questions, et couplent les approches géomorphologiques, palynologiques, malacologiques et archéologiques (Diot, 1991; Diot et al., 2002; Coquillas et al., 2006). Grâce à ces travaux, il est possible de restituer assez finement le paysage tel qu'il était à l'âge du Bronze moyen en Médoc.

Durant l'Holocène, le paysage médocain était fortement influencé par l'estuaire et l'océan Atlantique. L'analyse de la fluctuation du trait de côte depuis la Protohistoire est cruciale, mais nous ne disposons pas, à l'heure actuelle, d'une documentation suffisamment fiable pour la restituer (Pontee et al., 1998). Nous pouvons cependant proposer quelques grandes tendances générales à l'échelle de la façade atlantique. Entre 4000 et 1000 av. J.-C., la fluctuation du niveau marin et relativement faible. Des marais et des zones humides côtières se sont constitués progressivement le long de la façade atlantique. Entre 2500 et 1000 av. J.-C., de petites dunes côtières se sont formées. Elles sont néanmoins beaucoup plus petites que celles que nous pouvons observer aujourd'hui (Clavé, 2001, p. 65). La formation des zones humides est stoppée entre 1000 av. J.-C. et le début de notre ère. Des transgressions marines sont enregistrées sans que leur origine ne soit clairement identifiée. Ce phénomène a été observé dans différents marais du Nord et de l'Est du Médoc, tels que les marais de la Perge, de Talais ou de Reysson. De nombreuses analyses ont été conduites dans ces anciennes zones humides par différents chercheurs (Allen et al., 1970; Diot et Tastet, 1995). Des variations de la dynamique de ces marécages ont été observées durant l'âge du Bronze et l'âge du Fer. Au début de l'âge du Bronze, ces marais tidaux fonctionnaient majoritairement sous l'influence de l'océan. À partir du tout début du Bronze final et principalement durant l'âge du Fer, les zones humides vont progressivement se fermer aux influences marines et se combler. À partir de la fin de l'âge du Fer et durant l'époque romaine, les marais vont définitivement se combler, grâce notamment à la formation d'un cordon coquiller long de $25 \mathrm{~km}$ au nordest du Médoc, dénommé cordon de Richard. Sa largeur varie de 30 à $50 \mathrm{~m}$ et peut atteindre parfois $150 \mathrm{~m}$, pour une hauteur comprise entre quelques centimètres et $5 \mathrm{~m}$ (Tastet et al., 2000; Clavé, 2001, p. 165-180). Ce cordon est constitué de scrobicularia et se forme entre 1000 et le changement d'ère, période durant laquelle de nombreuses tempêtes se sont abattues sur le Médoc (Clavé, 2001, p. 177). Des dunes massives se sont mises en place à partir du Moyen Âge sur la côte, ce qui a eu pour effet de définitivement bloquer les influences marines et l'écoulement des eaux provenant des terres vers l'océan (Pontee et al., 1998). Ce rempart de sable a conduit à la formation des grands lacs d'eau douce actuels de Lacanau et d'Hourtin. Progressivement, durant les XVIII et XIX ${ }^{\mathrm{e}}$ siècles, ces dunes qui menaçaient les villages côtiers ont été stabilisées par l'implantation de forêts de pins. Actuellement les côtes du Nord du Médoc subissent d'importantes phases érosives, notamment durant les grandes marées hivernales. Le trait de côté recule de plusieurs mètres par an, malgré d'importants moyens mis en place pour lutter contre ce phénomène. Cette érosion a pour effet de mettre au jour des niveaux argileux anciens sous le cordon dunaire, livrant au passage de nombreux vestiges archéologiques. À partir de 1628, les zones humides du Médoc ont été drainées, en partie grâce à la création de polders le long de l'estuaire, implantés directement sur le cordon de Richard. Ces espaces gagnés sur l'océan et l'estuaire sont dénommés localement palus, ou mattes pour les espaces drainés durant les XVII ${ }^{\mathrm{e}}$ et XVIII ${ }^{\mathrm{e}}$ siècles (Coquillas, 2001). Cette modification du territoire est visible sur les cartes géologiques mais également cadastrales. Les lots divisés des terres asséchées sont réguliers, à l'inverse des terres initialement émergées. Ces cartes permettent de tracer le contour précis des anciens marais et sont de véritables supports pour l'analyse spatiale (fig. 2).

Cinq carottes ont été prélevées dans le marais de la Perge dans le Nord du Médoc, et plusieurs analyses palynologiques et géomorphologiques y ont été réalisées (Diot et al., 2002). Une des carottes donne une bonne illustration du paysage ancien dans ce secteur, où plusieurs phases de l'évolution de l'environnement ont été observées (fig. 3). Pour notre cadre chronologique de recherche, les enregistrements géomorphologiques et polliniques démontrent l'existence de marais tidaux dans l'Est du Médoc entre 3300 et 1100 av. J.-C. comme en témoignent les Dinoflagellata et de Chenopodiaceae. Entre 1100 et 100 av. J.-C., plusieurs enregistrements démontrent la disparition de l'Alnus et une importante croissance du Carex, qui témoigne de la montée des eaux des nappes phréatiques. Ce phénomène traduit le basculement du fonctionnement des marais avec une influence plus marquée de l'estuaire. Mais ces zones humides vont rapidement se transformer en marais saumâtres, comme en témoigne la carotte 9204 (fig. 3), avec l'apparition du Dinophyta et la prédominance du Chenopodiaceae. Entre 100 av. J.-C. et 700 apr. J.-C., la partie est du marais de la Perge enregistre le Carex et l'Ericaceae, témoins de la fermeture complète des marais aux influences marines et estuariennes et d'un fonctionnement plutôt continental. Ces différentes données témoignent d'une humidification importante du milieu à partir du Bronze final et une recrudescence des tempêtes, contemporaines de la formation du cordon de Richard (Diot et al. , 2002, p. 168).

Nous verrons par la suite que ce changement important du milieu correspond à une quasi-disparition des découvertes relatives au Bronze final. Avant d'aborder ce thème, il est nécessaire de proposer une synthèse sur la 


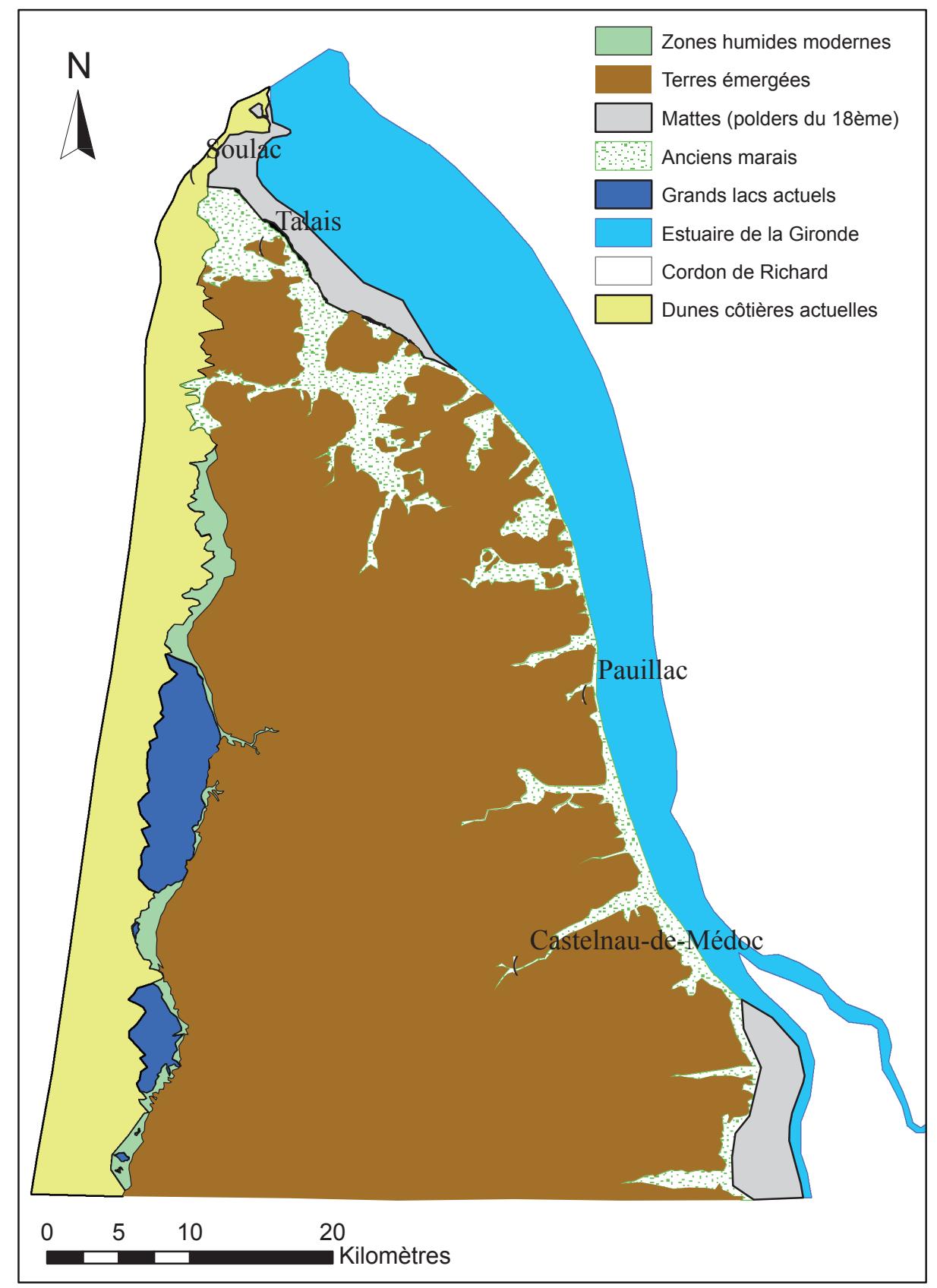

Fig. 2 - Reconstitution théorique des paysages du Médoc à partir du cadastre, de la carte géologique, des vues aériennes et des données paléoenvironnementales (SIG F. Couderc).

Fig. 2 - Theoretical reconstruction of the Médoc landscape using the land register, geological maps, aerial views and palaeo-environmental data (GIS F. Couderc).

composition matérielle des dépôts du Bronze moyen 2, en reprenant notamment les travaux sur l'Aquitaine de Céline Lagarde-Cardona (Lagarde-Cardona, 2012), et en apportant un regard synthétique sur les productions du Bronze moyen 2 en Médoc. Nous verrons que les dépôts métalliques médocains sont très homogènes, comme pour le reste de la façade atlantique pour la même période, en témoignent les travaux relatifs à la Bretagne ou à la Normandie (Gabillot, 2003; Fily, 2008) et au Centre-Ouest (Pautreau, 1979, Gomez de Soto, 1980). Mais le type et la localisation des dépôts du Médoc en font un cas particulier pour le domaine atlantique.

\section{LA QUESTION DE LA CHRONOLOGIE DES DÉPÔTS}

Tl est difficile d'identifier avec précision la chronologie d'un dépôt. Les typologies des objets métalliques varient peu au cours du Bronze moyen, et certains ensembles récents peuvent contenir des objets typologiquement plus anciens. De plus, les vases contenant certains des dépôts qui pourraient nous renseigner sur la chronologie de l'ensemble sont rarement documentés ou conservés. André Coffyn avait proposé trois 

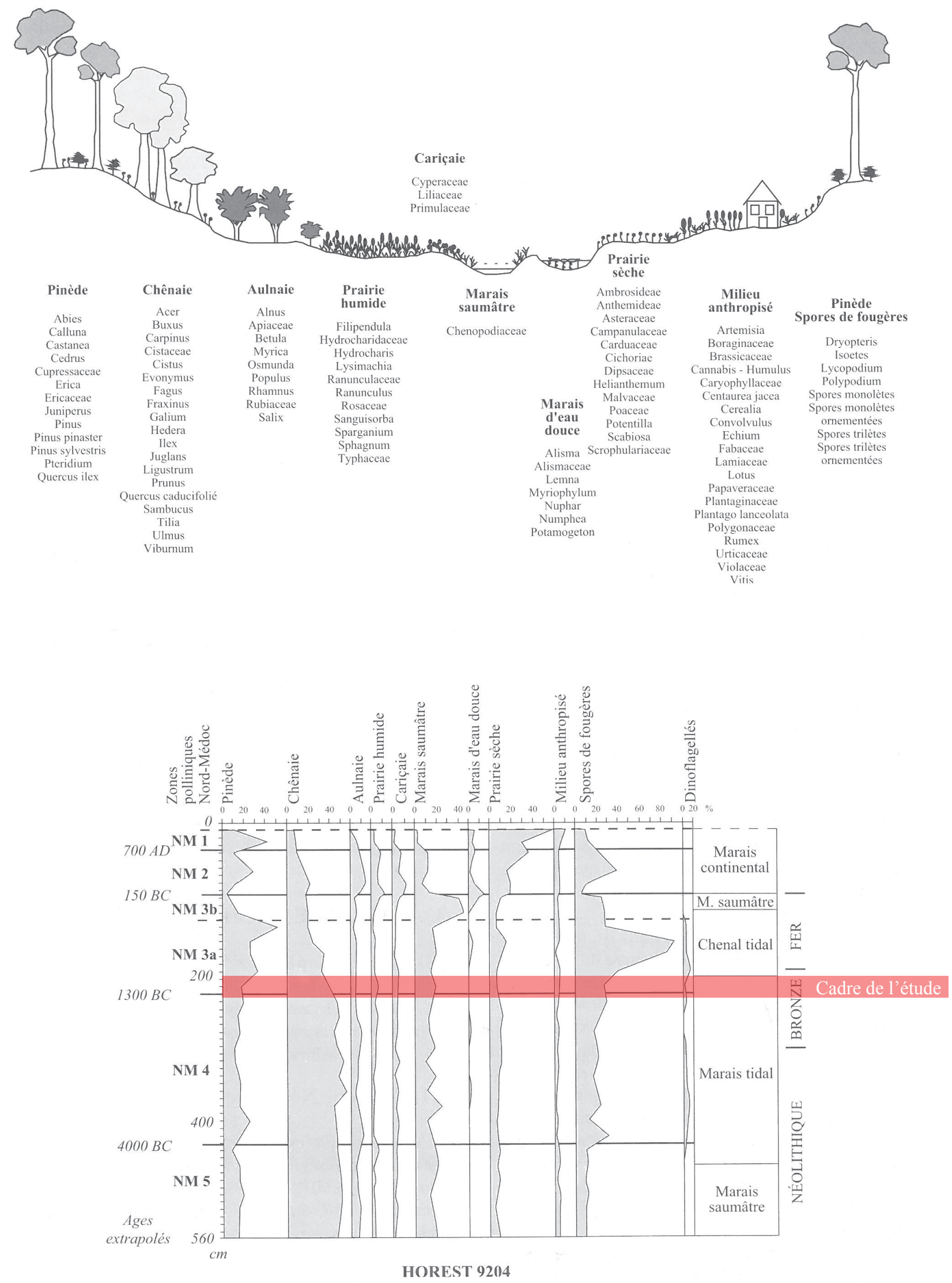

Fig. 3 - Zonation environnementale des assemblages végétaux des régions littorales de la côte atlantique et diagramme pollinique (carotte 9204) du marais de la Perge (d'après Diot et al., 2002; Coquillas et al., 2006).

Fig. 3 - Environmental zoning of the vegetation in Atlantic coastal regions and pollen diagram (core samples 9204) of La Perge marshland (after Diot and al., 2002; Coquillas et al., 2006). 
phases successives pour le Bronze moyen médocain à partir de la typologie des haches et de leur association avec d'autres objets dans les dépôts (Coffyn, 1972). La sériation des dépôts réalisée par Céline LagardeCardona est plus convaincante pour proposer un phasage des dépôts d'Aquitaine et du Médoc (LagardeCardona, 2012, p. 89-91). Seul le dépôt du Castel à Castelnau-de-Médoc peut être attribué au Bronze moyen 1 en Médoc avec les deux haches du type Martillac. Le deuxième horizon proposé par Céline LagardeCardona se caractérise par l'association des haches du type médocain avec le type Centre-Ouest (relativement rare en Médoc). Ces ensembles se situeraient entre l'extrême fin du Bronze moyen 1 et plus probablement durant le Bronze moyen 2. Le troisième horizon voit apparaître des associations plus hétéroclites avec les haches du type breton, vendéen et normand, ainsi que les petites haches médocaines. Cet horizon daterait principalement du Bronze moyen 2, voire du tout début du Bronze final 1 pour les dépôts les plus complexes dont la part des haches dans l'ensemble diminue au profit d'autres classes fonctionnelles. Cependant les dépôts présentant une part moindre de haches sont très minoritaires en Médoc.

La pratique des dépôts en Médoc semble donc se concentrer principalement sur le Bronze moyen 2, bien que quelques ensembles peuvent être plus anciens ou un peu plus récents. Nous parlerons donc dans cet article d'un Bronze moyen 2 au sens large, qui peut renfermer quelques nuances, mais l'état actuel des connaissances ne nous permet pas d'affiner plus précisément la chronologie.

\section{TYPES ET COMPOSITIONS DES DÉPÔTS DU BRONZE MOYEN MÉDOCAIN}

\section{Pondération et quantification des objets dans les dépôts médocains}

En Médoc, ce sont au total cinquante-sept dépôts qui sont recensés (soit plus de la moitié des dépôts aquitains) pour le Bronze moyen 2 (1450-1250 av. J.-C.). Ils regroupent 1057 haches, soixante et onze éléments de parures, vingtcinq déchets de métallurgie, quatre éléments d'armement, trois outils et trois fragments de vaisselle en bronze. Les études anciennes renseignent rarement la masse de chaque objet (tabl. 1), mais nous pouvons, pour un certain nombre des dépôts les plus récemment découverts, proposer une masse globale de l'ensemble. Les dépôts médocains sont lourds, avec une majorité d'entre eux pesant entre 7 et $11 \mathrm{~kg}$. Concernant le nombre des objets contenus, il est assez variable, entre sept et vingt-deux par dépôt (fig. 4). Si nous considérons uniquement la masse des haches découvertes en Médoc, elle représente au total près de $660 \mathrm{~kg}$ de métal. Cette quantité est considérable dans une microrégion telle que le Médoc, bien que durant le Bronze moyen 2, les autres régions de la façade atlantique regroupent elles aussi des quantités importantes d'objets métalliques (Galinand 2008)

\section{Classifications des dépôts médocains}

Quatre types de composition des dépôts sont identifiables en Médoc (tabl. 2). Quarante-trois sont composés

\begin{tabular}{|l|c|c|c|}
\hline Dépôt & Nombre d'objets & Masse totale (en kg) & Objets non pesés \\
\hline Padarnac (Pauillac) & 29 & 19,1 & - \\
\hline Chemin-Profond (Lesparre) & 27 & 18,5 & - \\
\hline L'Amélie VII (Soulac) & 24 & 15,3 & 2 haches à rebords \\
\hline Le Chalet II (Saint-Germain-d'Esteuil) & 25 & 15,2 & - \\
\hline Le Chalet I (Saint-Germain-d'Esteuil) & 50 & 14,6 & - \\
\hline Le Pouyalet II (Pauillac) & 16 & 10,8 & - \\
\hline L'Amélie IV (Soulac) & 18 & 10,1 & - \\
\hline L'Amélie V (Soulac) & 11 & 8,2 & - \\
\hline L'Amélie VI (Soulac) & 10 & 7,5 & - \\
\hline Le Lazaret de Trompeloup (Pauillac) & 10 & 7,1 & 4 bracelets \\
\hline L'Amélie I (Soulac) & 8 & 4,2 & - \\
\hline Montalivet I (Vendays-Montalivet) & 24 & 4,0 & - \\
\hline Le Bourg (Pauillac) & 4 & 2,6 & - \\
\hline Montalivet II (Vendays-Montalivet) & 7 & 2,0 & - \\
\hline L'Amélie VIII (Soulac) & 13 & 1,5 & - \\
\hline L'Amélie III (Soulac) & 3 & 1,0 & - \\
\hline
\end{tabular}

Tabl. 1 - Nombre d'objets et masse totale des objets des dépôts médocains bien documentés.

Table 1 - Number of objects and total weight of objects in the well-documented hoards in Médoc. 


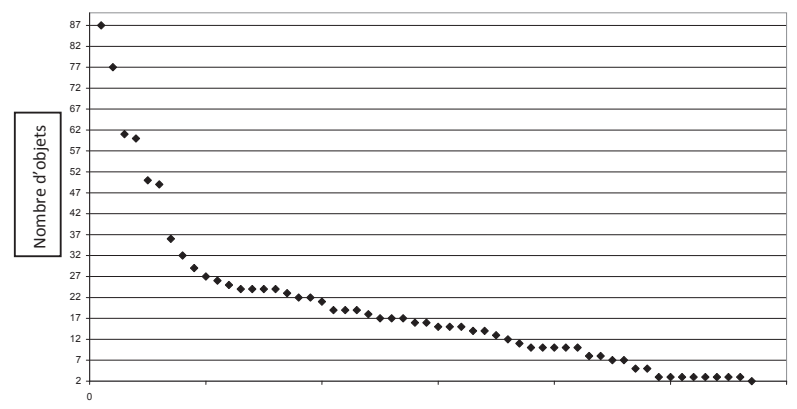

Fig. 4 - Diagramme du nombre d'objets contenus dans les dépôts.

Fig. 4 -Diagram illustrating the number of objects found in the hoards.

uniquement par des haches, neuf par des haches et un second type d'objet (dépôts à double composante), deux par quatre ou cinq types d'objets (dépôts complexes) et trois dépôts sont considérés comme particulier car la part des haches dans leur composition est insignifiante (aucune hache ou moins de $15 \%$ de l'ensemble). Les dépôts complexes ou particuliers où la part des parures prend de l'importance pourraient plutôt se rattacher à l'extrême fin du Bronze moyen 2 ou au Bronze final 1 ancien.

Ainsi, les haches représentent la production métallique la plus importante du Bronze moyen en Médoc qui nous soit parvenue. Il est possible d'identifier les types de haches pour vingt dépôts seulement (tabl. 3). Pour ces dépôts bien documentés, nous constatons que les haches de type médocain dominent largement le corpus, mais elles sont régulièrement associées à d'autres types (breton, Centre-Ouest). Pour les autres dépôts dont la documentation est médiocre, nous avons parfois des informations sur le type de quelques-unes des haches qui composent l'ensemble (tabl. 4). Dans ce cas, la part des productions du type médocain est également très importante, suivie par les haches du type breton. Mais nous ne connaissons pas le type de la majorité des autres haches, nous savons seulement que les haches à rebords représentent le double de celles à talon (fig. 5). Il est intéressant de remarquer que trois dépôts contiennent uniquement des haches à talon. C'est le cas du dépôt de Château Langoa (SaintJulien-Beychevelle), avec vingt-six haches, le dépôt de Rouman III (Gaillan-en-Médoc) avec quatorze haches et le dépôt de l'Aygua III (Bégadan) avec vingt haches. Malheureusement, ces objets ont disparu anciennement et nous ne pouvons donc pas identifier leur type.

Certains dépôts contiennent des objets fragmentés. Pour les découvertes anciennes, les premiers auteurs ont très rarement décrit ou même conservé ces éléments qui présentaient peu d'intérêt à leurs yeux. Ceci nous prive d'une précieuse documentation. De la parure et des haches sont fragmentées dans le dépôt de l'Aygua I (Bégadan), dans le dépôt du Chalet I (Saint-Germain-d'Esteui) et dans le dépôt de Montalivet I (Vendays-Montalivet). Les objets

\begin{tabular}{|l|c|c|c|c|}
\hline Dépôts à double composante & Hache & Parure & Déchet métallique & Outil \\
\hline Aygua I (Bégadan) & 82 & - & 5 & - \\
\hline Corconnac (Saint-Laurent-Médoc) & 14 & - & 1 & - \\
\hline Le Vignaud (Talais) & 21 & 2 & - & - \\
\hline L'Amélie I (Soulac-sur-Mer) & 7 & - & 1 & - \\
\hline Montalivet II (Vendays-Montalivet) & 6 & - & 1 & - \\
\hline L'Amélie III (Soulac-sur-Mer) & 2 & - & 1 & - \\
\hline Castel (Castelnau-Médoc) & 2 & - & & 1 \\
\hline Montalivet I (Vendays-Montalivet) & 11 & 8 & - & - \\
\hline Château Léoville (Saint-Julien-Beychevelle) & 46 & 3 & - & - \\
\hline
\end{tabular}

\begin{tabular}{|l|c|c|c|c|c|}
\hline Dépôts complexes & Hache & Parure & Outil & Armement & Déchet métallique \\
\hline Le Chalet I (Saint-Germain-d'Esteuil) & 33 & 9 & 1 & 2 & 5 \\
\hline Le Temple I (Saint-Vivien-Médoc) & 18 & 4 & 1 & 1 & - \\
\hline
\end{tabular}

\begin{tabular}{|l|c|c|c|c|c|}
\hline Dépôts exceptionnels & Hache & Parure & Déchet métallique & Armement & Vaisselle \\
\hline L’Amélie VIII (Soulac-sur-Mer) & 2 & 9 & 1 & 1 & - \\
\hline L'Amélie II (Soulac-sur-Mer) & 2 & 1 & 10 & - & 3 \\
\hline Le Pouyalet III (Pauillac) & - & 36 & - & & - \\
\hline
\end{tabular}

Tabl. 2 - Présentation de la composition des dépôts des ensembles à double composante, complexes et particuliers, ainsi que de la catégorie fonctionnelle des objets.

Table 2 - Presentation of the contents of the hoards (double components, complex and specific), and the usage of objects. 


\begin{tabular}{|c|c|c|c|c|c|c|c|c|c|}
\hline Dépôt & 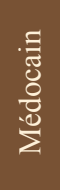 & 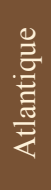 & 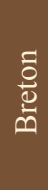 & $\begin{array}{l}\overrightarrow{0} \\
0 \\
0 \\
\vdots \\
0 \\
0 \\
000 \\
0\end{array}$ & 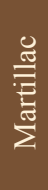 & $\begin{array}{l}\text { Z्ञ } \\
\text { Е } \\
\text { Z } \\
\text { Z }\end{array}$ & 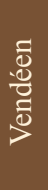 & $\begin{array}{l}\text { हే } \\
\text { ठ } \\
\text { ठ }\end{array}$ & 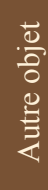 \\
\hline Chemin-Profond (Lesparre) & 24 & - & - & 1 & - & - & - & 2 & - \\
\hline Le Chalet II (Saint-Germain) & 20 & - & - & 1 & - & - & 4 & - & - \\
\hline L'Amélie VII (Soulac) & 21 & - & - & 3 & - & - & - & - & - \\
\hline Abbaye de l'île (Ordonnac) & 12 & - & 4 & 1 & - & - & - & 2 & - \\
\hline L'Amélie VIII & 1 & - & 1 & - & - & - & - & - & 11 \\
\hline Le Temple I (Saint-Vivien) & 11 & - & 3 & - & - & 1 & 2 & 1 & 6 \\
\hline L'Amélie I (Soulac) & 5 & - & 1 & - & - & - & - & - & 1 \\
\hline Le Bourg (Pauillac) & 4 & 1 & - & - & - & - & - & - & - \\
\hline L'Amélie IV (Soulac) & 17 & 1 & - & - & - & - & - & - & - \\
\hline Padarnac (Pauillac) & 28 & - & - & - & - & - & - & 1 & - \\
\hline La Gravette (Lesparre) & 3 & - & - & - & - & - & - & - & - \\
\hline Saint-Laurent I & 3 & - & - & - & - & - & - & - & - \\
\hline Le Pouyalet II (Pauillac) & 16 & - & - & - & - & - & - & - & - \\
\hline L’Amélie III (Soulac) & 2 & - & - & - & - & - & - & - & 1 \\
\hline Le Bourg (Saint-Estéphe) & 7 & - & - & - & - & - & - & - & - \\
\hline L'Amélie V (Soulac) & 11 & - & - & - & - & - & - & - & - \\
\hline L’Amélie VI (Soulac) & 10 & - & - & - & - & - & - & - & - \\
\hline Le Pouyalet I (Pauillac) & 21 & - & - & - & - & - & - & - & - \\
\hline Le Lazaret de Trompeloup (Pauillac) & 10 & - & - & - & - & - & - & - & - \\
\hline Castel (Castelnau-Médoc) & - & - & - & - & 2 & - & - & - & 1 \\
\hline
\end{tabular}

Tabl. 3 - Types des haches dans les vingt dépôts bien documentés.

Table 3 - Axe types for the twenty well-documented hoards.

\begin{tabular}{|c|c|c|c|c|c|c|c|c|}
\hline Médocain & Breton & Vendéen & Centre-Ouest & Normand & Martillac & Werrar & Haguenau & Inconnu \\
\hline 70 & 28 & 6 & 4 & 3 & 2 & 1 & 1 & 647 \\
\hline
\end{tabular}

Tabl. 4 - Présentation des types de quelques haches connues dans les trente-sept dépôts mal documentés N.B : la part des indéterminés est très importante dans ces ensembles mais la famille est parfois connue (cf. fig. 5).

Table 4 - Presentation of some of the known axe types in the thirty-seven poorly documented hoards N.B: The number of undetermined types is high in those hoards, though the family is sometimes known (cf. fig. 5).

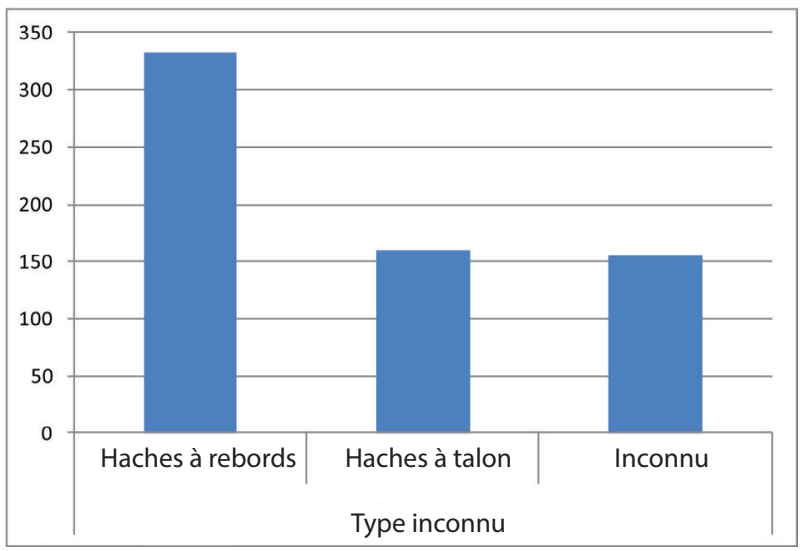

Fig. 5 - Histogramme de la famille des 647 haches de type imprécis.

Fig. 5 - Histogram of the 647 axes of unclear classification. dans les dépôts médocains présentent des états de finition et des traces d'usage très variés. Certains sont bruts de coulée, d'autres ont subi un premier traitement post-coulée et parfois semblent avoir été utilisés. Nous renvoyons aux travaux de Céline Lagarde qui a largement traité de ces questions (Lagarde-Cardona, 2012, p. 122-137).

\section{Modalités d'enfouissement}

Les conditions d'enfouissement ont rarement été examinées lors des découvertes anciennes, voire même récentes, car il s'agit quasi-exclusivement de trouvailles fortuites. Dans certains cas, les auteurs du $\mathrm{XIX}^{\mathrm{e}}$ siècle signalent qu'un vase contenait les objets. Ils les décrivent parfois avec des «pustules » d'argile qui recouvraient la céramique, comme pour le dépôt du Vignaud à Talais 


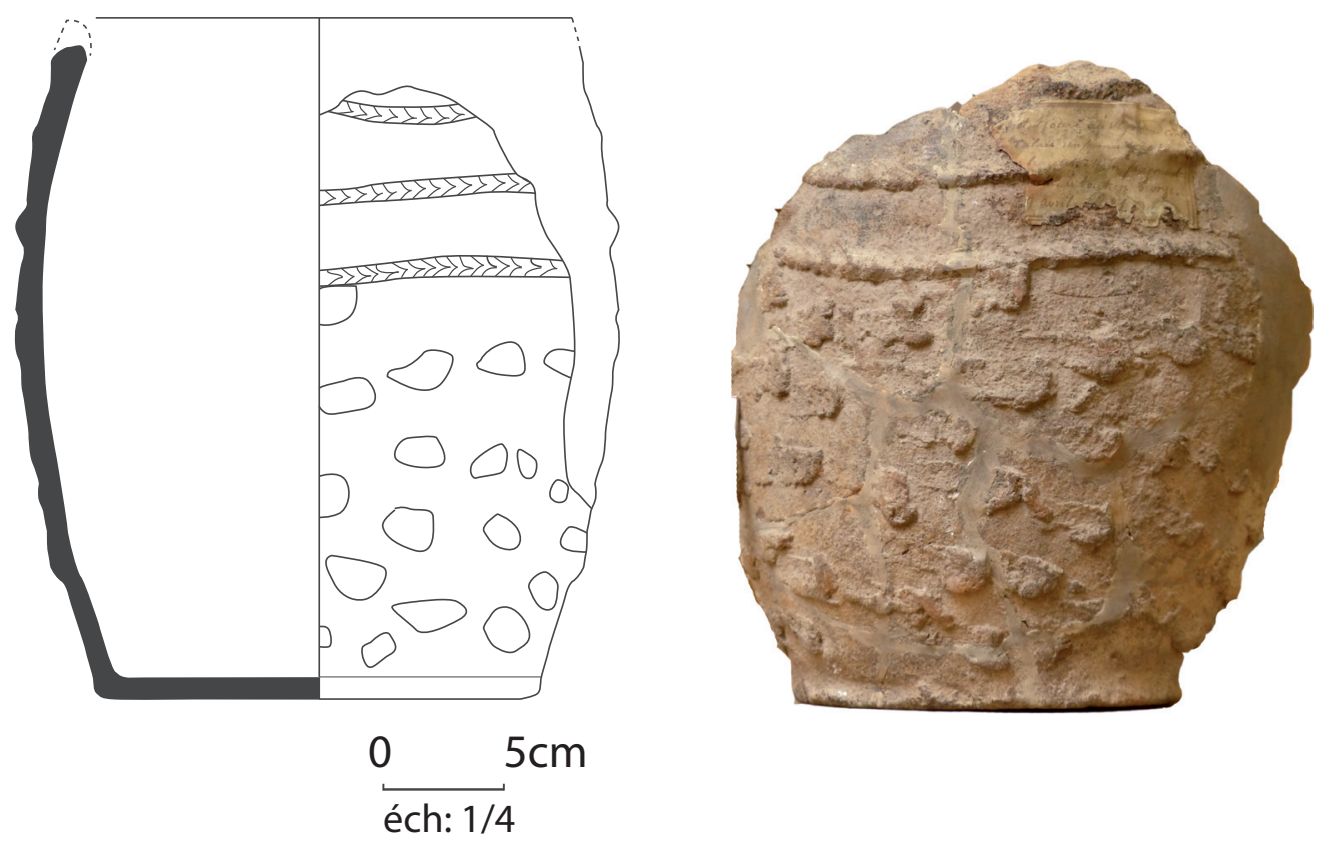

Fig. 6 - Vase du dépôt des Vignaud à Talais conservé au musée d'Aquitaine à Bordeaux (DAO F. Couderc).

Fig. 6 - Vase from the Vignaud hoard, Talais, in the Musée d'Aquitaine of Bordeaux (CAD F. Couderc).

(Meynieu, 1881). Ces vases à pastillage sont des productions communes du Bronze moyen en Aquitaine (fig. 6). Vingt-cinq dépôts ont été découverts dans des vases, mais la plupart des céramiques ont aujourd'hui disparu. Nous savons cependant avec certitude qu'au moins huit d'entre eux sont des vases à pastillage. Un soin particulier est parfois apporté à la disposition des objets, comme pour le dépôt du Chalet I à Saint-Germain-d'Esteuil, où les tranchants étaient tous orientés vers le haut (RoussotLarroque, 1999). Une découverte très révélatrice des comportements a été faite sur la plage de l'Amélie à Soulac-sur-Mer. Cinq dépôts ont été trouvés successivement à quelques mètres les uns des autres, suite aux grandes marées hivernales (Coffyn et al., 1995). Tous ont été découverts dans des vases ou associés à de la céramique à pastillage. Ils contenaient au total soixantedouze haches et $7 \mathrm{~kg}$ de fragments métalliques, ce qui représente une masse totale de $53,76 \mathrm{~kg}$ de métal. Un de ces dépôts renfermait le seul objet en or connu en Médoc pour le Bronze moyen, composé de fils enroulés (fig. 7). Une autre concentration des dépôts sur un même espace est attestée autour de Pauillac dans un rayon d'un kilomètre, où plusieurs dépôts ont été découverts durant le $\mathrm{XIX}^{\mathrm{e}}$ siècle (à Padarnac, au Pouyalet, au Lazaret et au bourg). La récurrence des dépôts dans une même zone, semble être l'un des marqueurs de cette pratique par les sociétés du Bronze moyen 2 en Médoc. Nous ne pouvons pas mesurer précisément le laps de temps qui s'est écoulé entre l'enfouissement de chacun des dépôts, mais l'utilisation régulière d'un même type de contenant (vase en tonnelet parfois associé à du pastillage), leur proximité et l'homogénéité de leur composition laissent présumer que tout au plus, quelques années voire quelques mois seulement séparent l'enfouissement de chacun de ces dépôts.

\section{Analyse typologique et de distribution des haches du Bronze moyen en Médoc et en Aquitaine}

Au total, quatre-vingt-dix-huit dépôts terrestres au moins ont été retrouvés en Aquitaine et ils contenaient un minimum de 1559 objets. Quarante-six découvertes au moins de bronzes sont dites " isolées » et représentent au total cinquante-huit objets. Les contextes domestiques et funéraires sont mal documentés en Aquitaine, surtout pour sa partie septentrionale avec seulement quinze objets métalliques en contexte domestique et douze objets répartis entre sept sites à vocation funéraire. Nous ne connaissons pas les contextes de 111 découvertes qui regroupent au total 137 objets (Lagarde-Cardona, 2012).

Ce sont principalement des haches qui ont été découvertes avec 1571 exemplaires au moins pour l'Aquitaine.

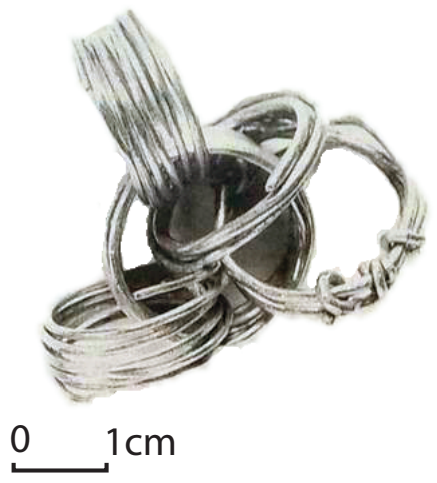

Fig. 7 - Fils d'or enroulés en anneaux du dépôt de L'Amélie III (d'après Coffyn et al., 1995).

Fig. 7 - Rolls of gold wire from L'Amélie III hoard (from Coffyn et al., 1995). 
La parure arrive en seconde position, mais loin derrière les haches avec quelques 108 bracelets, deux épingles et un rasoir. Quarante éléments sont classés comme des déchets métalliques, trente-quatre comme éléments d'armement, douze objets sont classifiés comme des outils et trois éléments sont de la vaisselle en bronze. La prépondérance des haches dans le corpus métallique du Bronze moyen en Aquitaine est caractéristique de la période, surtout pour la phase la plus récente, le Bronze moyen 2, entre environ 1450 et 1250 av. J.-C (fig. 8).

La prépondérance des haches en Médoc n'est pas exceptionnelle. Cette production reste la mieux documentée pour tout l'âge du Bronze moyen en Europe occidentale (Fontijn, 2002). Concernant les haches, nous n'aborderons pas les questions des variations morphométriques des différents types, qui peuvent refléter des origines de production différentes, voire mettre en évidence des productions locales inspirées de types exogènes (Gabillot et al., 2017). En Aquitaine nous pouvons identifier trois grandes origines principales (fig. 9). Il y a tout d'abord les productions locales. Elles sont identifiées à partir des aires de distribution, mais également de quelques rares moules découverts à proximité du site de la Lède du Gurp à Grayan-et-l'Hôpital (Frugier et al., 1983; RoussotLarroque, 1997) Ces productions locales sont des haches à rebords des types médocain, Thonac-Vanxains, libournais et de Martillac. Il y a également des productions d'origine atlantique, mais qui pourraient aussi être des productions locales inspirées de types atlantiques, avec les haches à rebords du type vendéen et les haches à talon des types breton et normand (Coffyn, 1972; Mélin, 2014). Les productions ou inspirations continentales sont plus rares et sont attestées par des haches à talon du type du Centre-Ouest. Il est intéressant de remarquer que les productions les plus massives sont les haches du type médocain, avec des objets pouvant atteindre $20 \mathrm{~cm}$ de long pour $800 \mathrm{~g}$. Les haches du type de Martillac sont également assez imposantes avec des individus mesurant $16 \mathrm{~cm}$ de long pour environ $600 \mathrm{~g}$. Ces productions dites « locales » sont réellement massives en comparaison des productions d'inspiration continentale et atlantique de haches à talon mesurant rarement plus de 15 à $18 \mathrm{~cm}$ de long pour un poids avoisinant les 300 à $500 \mathrm{~g}$. Les haches médocaines se retrouvent sur une aire de répartition très large, couvrant principalement tout le Sud-Ouest de la France. Il existe des variantes plus petites de ces haches médocaines (Lagarde-Cardona, 2012) avec des dimensions proches des haches à talon. Elles sont plus rares que les grandes et seraient produites surtout à la fin du Bronze moyen (Lagarde-Cardona, 2012, p. 91). Le diagramme proposé illustre parfaitement l'existence de deux groupes distincts (fig. 10).

La comparaison des aires de répartition des différents modèles de haches illustre que les types atlantiques sont comparativement plus présents en Médoc que dans le reste de l'Aquitaine et du Centre-Ouest de la France (fig. 11, 12 et 13). Les haches des types breton et vendéen sont bien représentées en Médoc tandis que le type $\mathrm{du}$ Centre-Ouest est rare, alors que son aire principale de diffusion est (comme pour les types breton et vendéen)

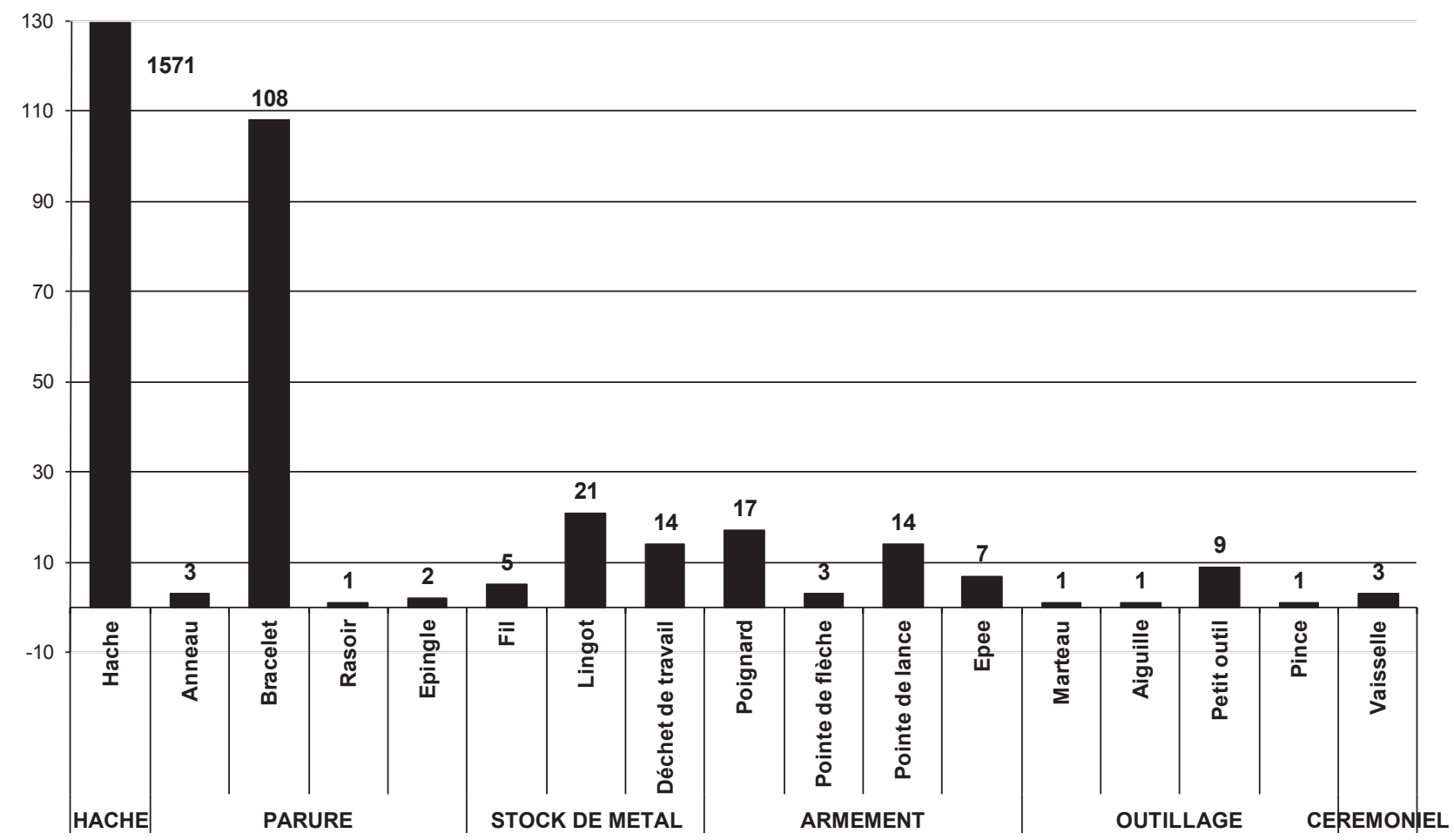

Fig. 8 - Histogramme des catégories fonctionnelles des objets métalliques du Bronze moyen retrouvés en Aquitaine (d'après LagardeCardona, 2012).

Fig. 8 - Histogram of usage of Middle Bronze Age metal objects from Aquitaine (after Lagarde-Cardona, 2012). 


\section{Productions de type local}

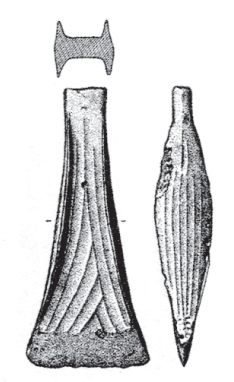

Thonac-Vanxains

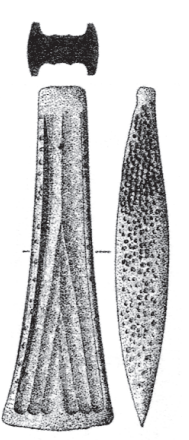

Libournais

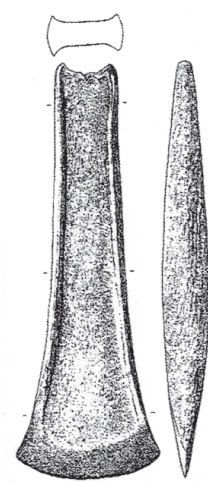

Martillac

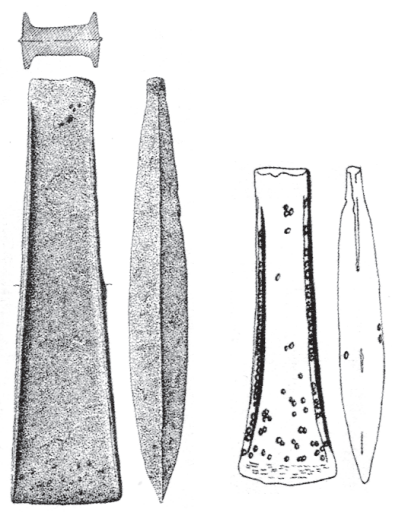

Médocaine $\left.\begin{array}{c}5 \mathrm{~cm} \\ {[} \\ 0\end{array}\right]$

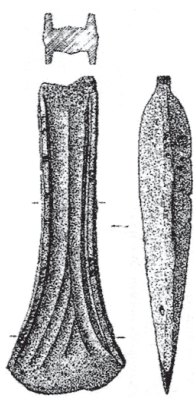

Vendéen

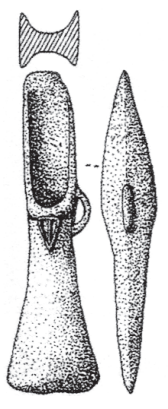

Normand

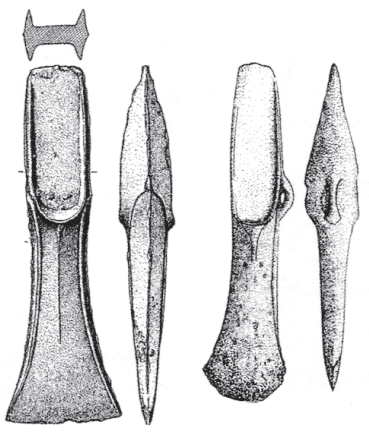

Breton

\section{Productions de type continental}

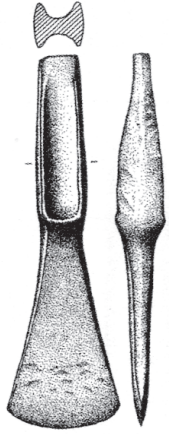

Orléans

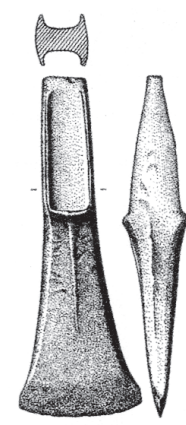

Centre-Ouest

Fig. 9 - Typologie des haches retrouvées dans les dépôts médocains (d'après Lagarde-Cardona, 2012).

Fig. 9 - Typology of axes from Médoc hoards (after Lagarde-Cardona, 2012). 


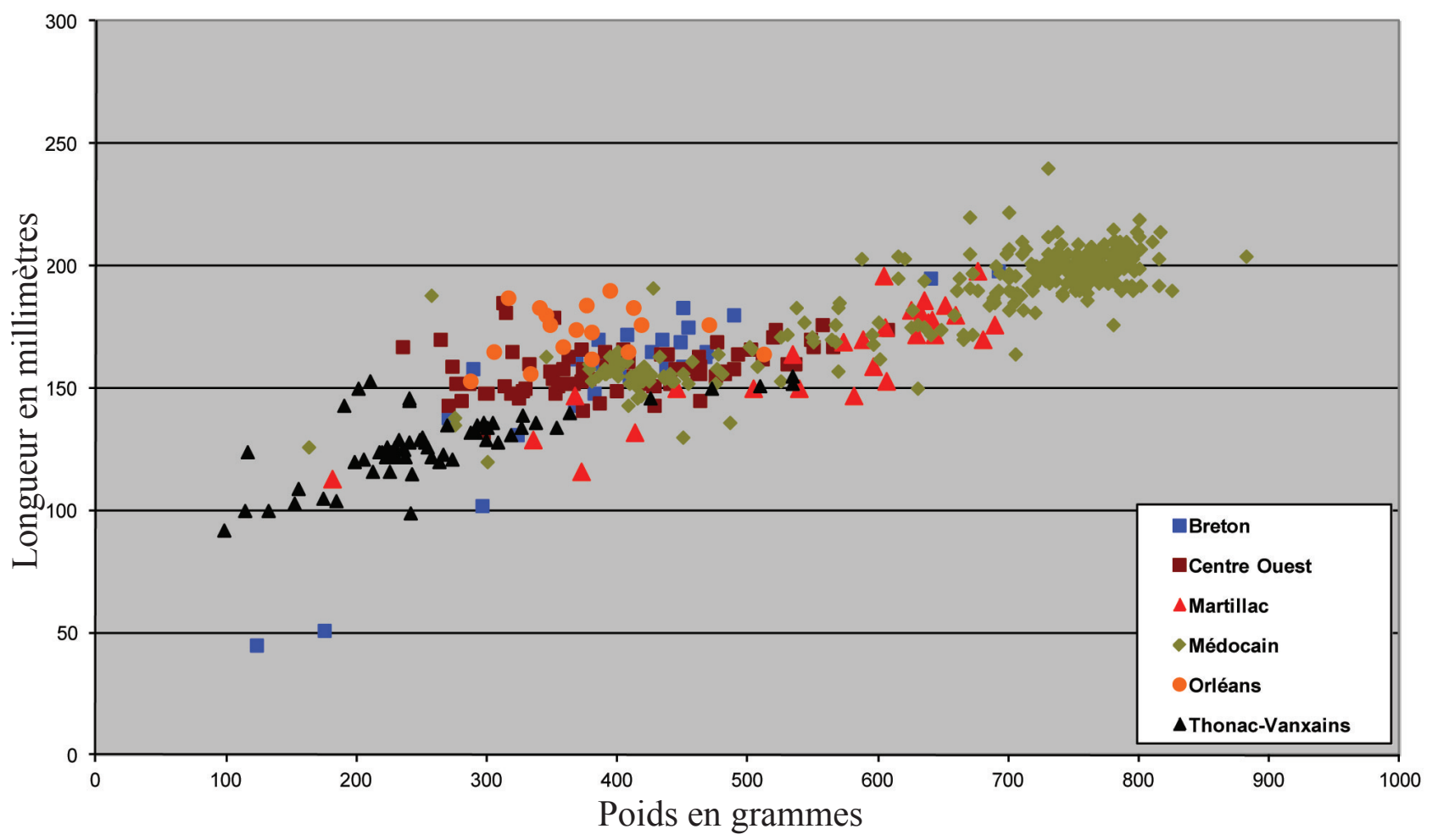

Fig. 10 - Diagramme du rapport longueur/masse des différents types de hache.

Fig. 10 - Diagram of weight/length ratio of different types of axes.

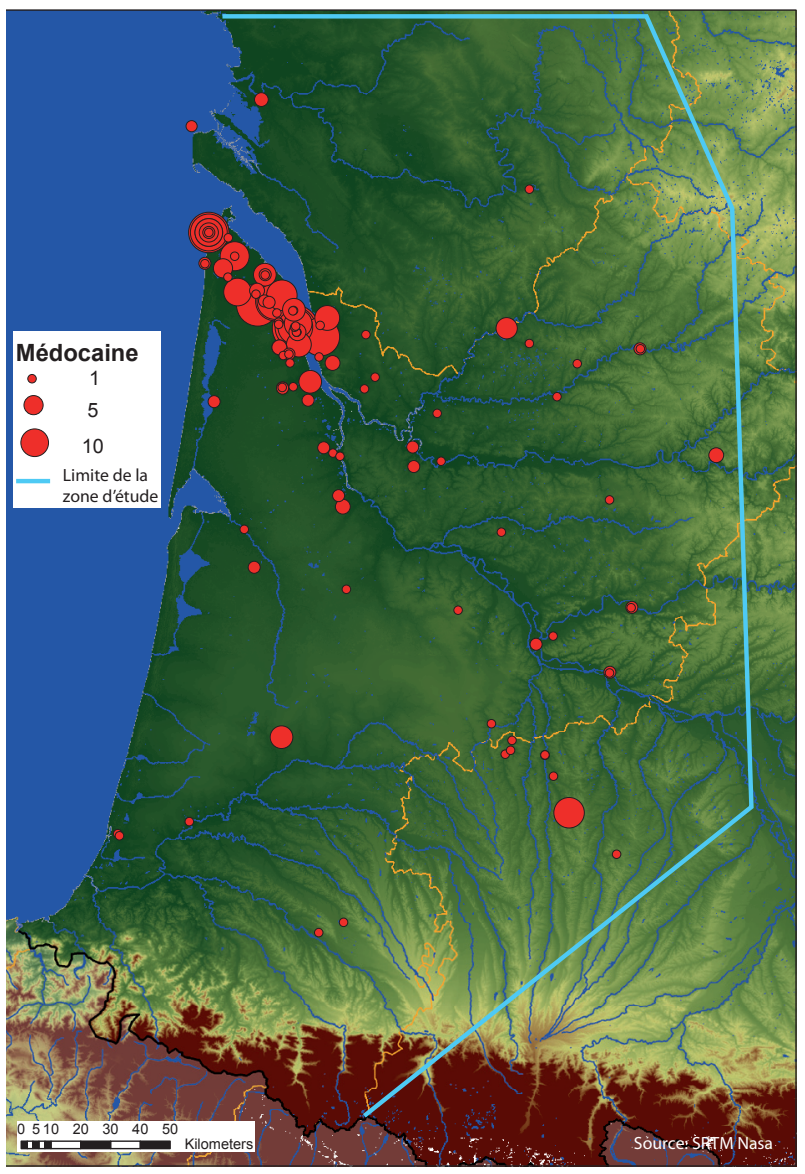

Fig. 11 - Carte de répartition des haches du type médocain en Aquitaine, dans le Centre-Ouest de la France et dans le Gers (SIG: F. Couderc; d'après Gomez de Soto, 1995 et LagardeCardona, 2012 complété).

Fig. 11 - Distribution map of Médoc type axes in Aquitaine, in the Centre-Ouest region of France and in the Gers (GIS F. Couderc; after Gomez de Soto, 1995 and Lagarde-Cardona, 2012 completed).

géographiquement très proche. La présence du type normand est plus exceptionnelle car son aire principale de répartition est beaucoup plus septentrionale. Cette distinction est particulièrement évocatrice des réseaux d'échange en place au Bronze moyen (fig. 14). Le Médoc regroupe en effet soixante-et-une haches de type atlantique, soit le double du reste de l'Aquitaine, tandis que seules douze haches d'inspiration continentale se retrouvent dans les dépôts médocains. En région Centre-Ouest, nous retrouvons ce même type de répartition, avec sur la côte une importante majorité des types atlantiques, tandis que dans les terres, les types continentaux sont largement dominants (Pautreau, 1979; Gomez de Soto, 1980 et 1995).

\section{SYNTHÈSE SUR LA COMPOSITION DES DÉPÔTS MÉDOCAINS}

Tous constatons, comme pour le reste de l'Europe occidentale à la même période, que les dépôts du 


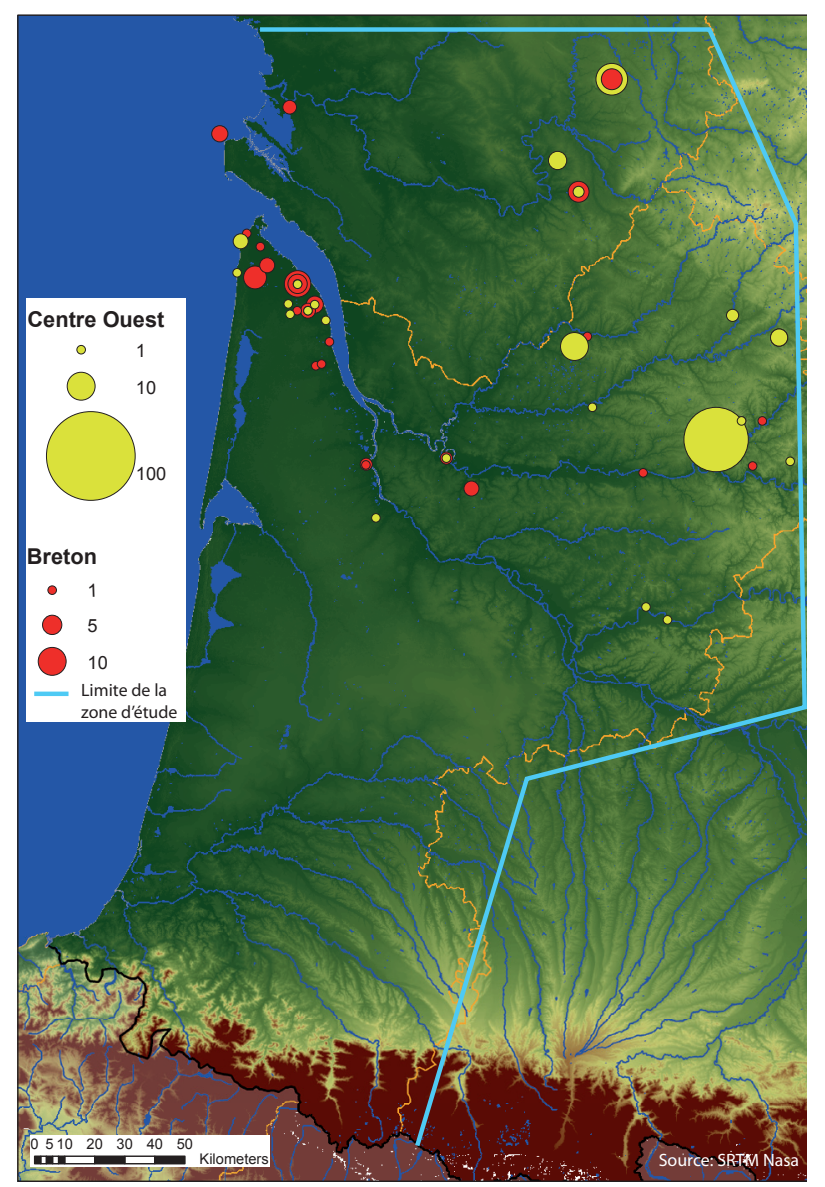

Fig. 12 - Carte de répartition des haches du type du CentreOuest et breton en Aquitaine et dans le Centre-Ouest de la France (SIG F. Couderc; d'après Gomez de Soto, 1995 et Lagarde-Cardona, 2012 complétés).

Fig. 12 - Distribution map of Centre-Ouest and Breton types axes in Aquitaine and in the the Centre-Ouest region (GIS F. Couderc; after Gomez de Soto, 1995 and Lagarde-Cardona, 2012 completed).

Bronze moyen en Médoc sont relativement homogènes dans leur composition et dans leur modalité d'enfouissement. Les haches sont prédominantes avec une majorité de types locaux. Ces dépôts datent pour l'essentiel $\mathrm{du}$ Bronze moyen 2, période où les contacts entre le Médoc et le reste du complexe atlantique semblent très importants, notamment pour s'approvisionner en produits métalliques et autres matières premières. Au Chalcolithique et au Bronze ancien, les objets métalliques en Médoc sont beaucoup plus rares. Seule la découverte de plus de 300 hachettes en cuivre sur la plage près de Vendays-Montalivet, démontre que des réseaux d'échanges importants étaient déjà en place à cette période, probablement avec d'autres régions du domaine atlantique. Ces hachettes mesurent entre 6 et $11 \mathrm{~cm}$ de long pour un poids de 8 à $15 \mathrm{~g}$ (fig. 15). Leur extrême finesse ne permet pas d'envisager une quelconque utilité fonctionnelle en l'état (Roussot-Larroque et al., 2001).

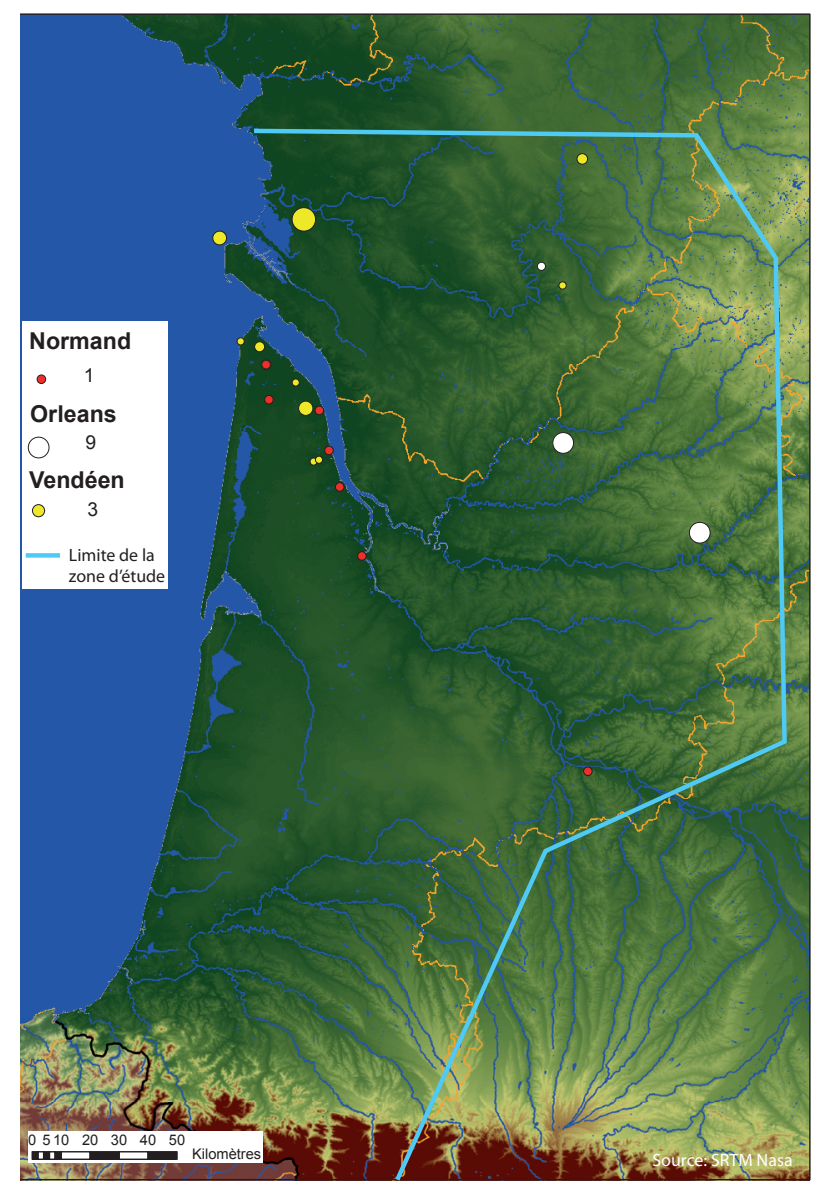

Fig. 13 - Carte de répartition des haches du type normand, vendéen et d'Orléans en Aquitaine et dans le Centre-Ouest de la France (SIG F. Couderc; d'après Gomez de Soto, 1995 et Lagarde-Cardona, 2012 complété).

Fig. 13 - Distribution map of Normand, Vendéen and Orléans types axes in Aquitaine and in the Centre-Ouest region (GIS F. Couderc; after Gomez de Soto, 1995 and Lagarde-Cardona, 2012 completed).

La place des échanges dans l'approvisionnement en produits métalliques est cruciale au Bronze moyen 2. Si certaines haches de type atlantique et continentale peuvent être des productions locales (Gabillot et al., 2017), l'appropriation de types exogènes témoigne au moins de contacts entre les différents grands groupes culturels. Mais ces relations et échanges étaient probablement aussi importants dans d'autres régions où la pratique des dépôts n'était pas aussi répandue qu'en Médoc. La fluctuation dans le temps de cette pratique témoigne de changements économiques mais également sociaux car, nous le verrons ensuite, cette pratique semble s'interrompre brutalement en Médoc au Bronze final et surtout durant le Bronze final 2 et 3 . Les causes de ces changements de comportement peuvent être multiples et seules des approches croisées permettent d'élargir les réflexions sur cette pratique. 


\section{Types des haches en Aquitaine}

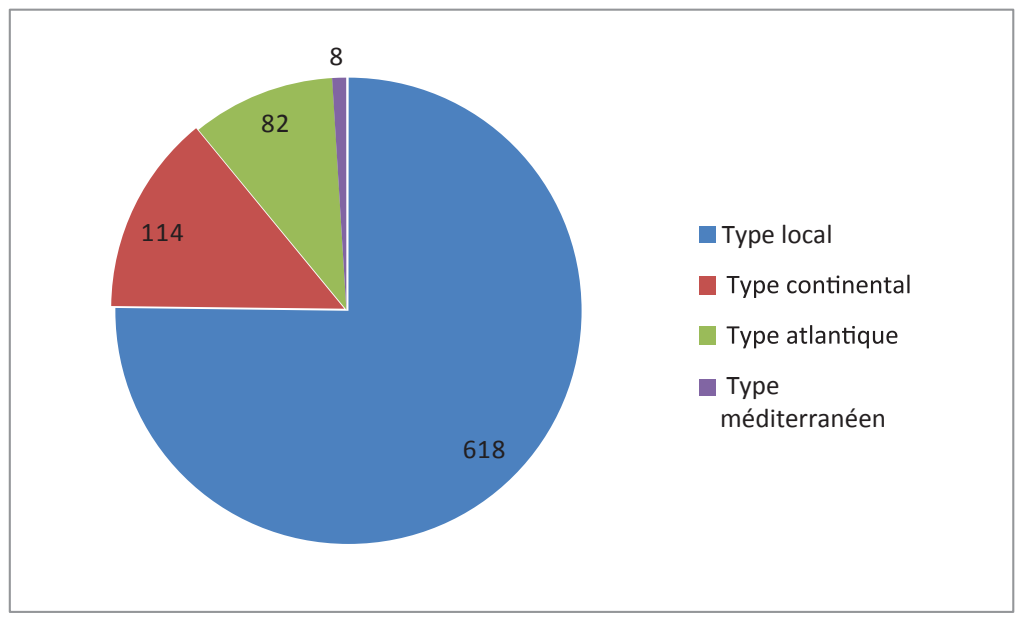

Types des haches en Médoc

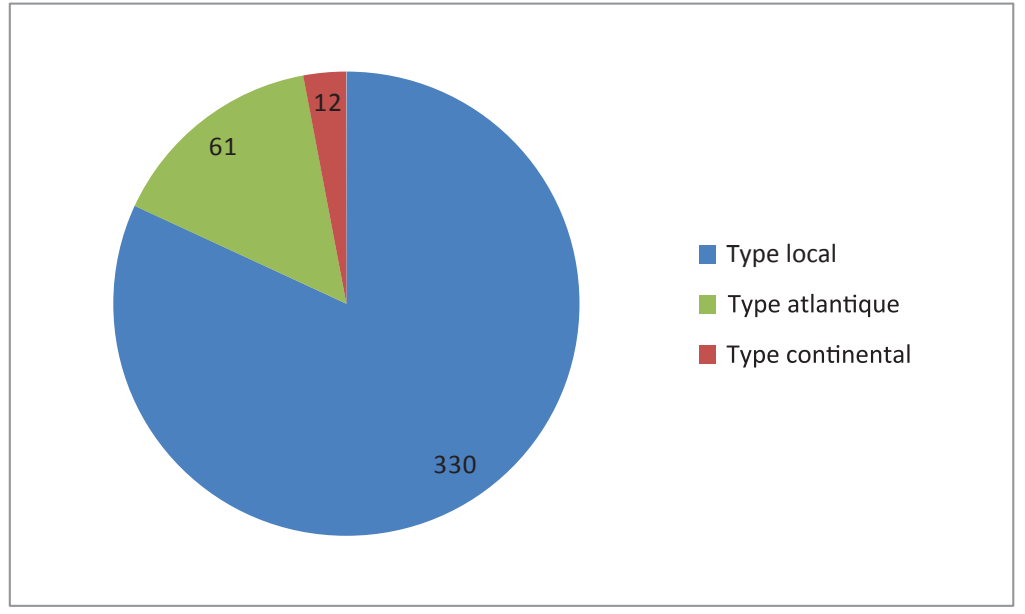

Nombre des haches par type en Médoc

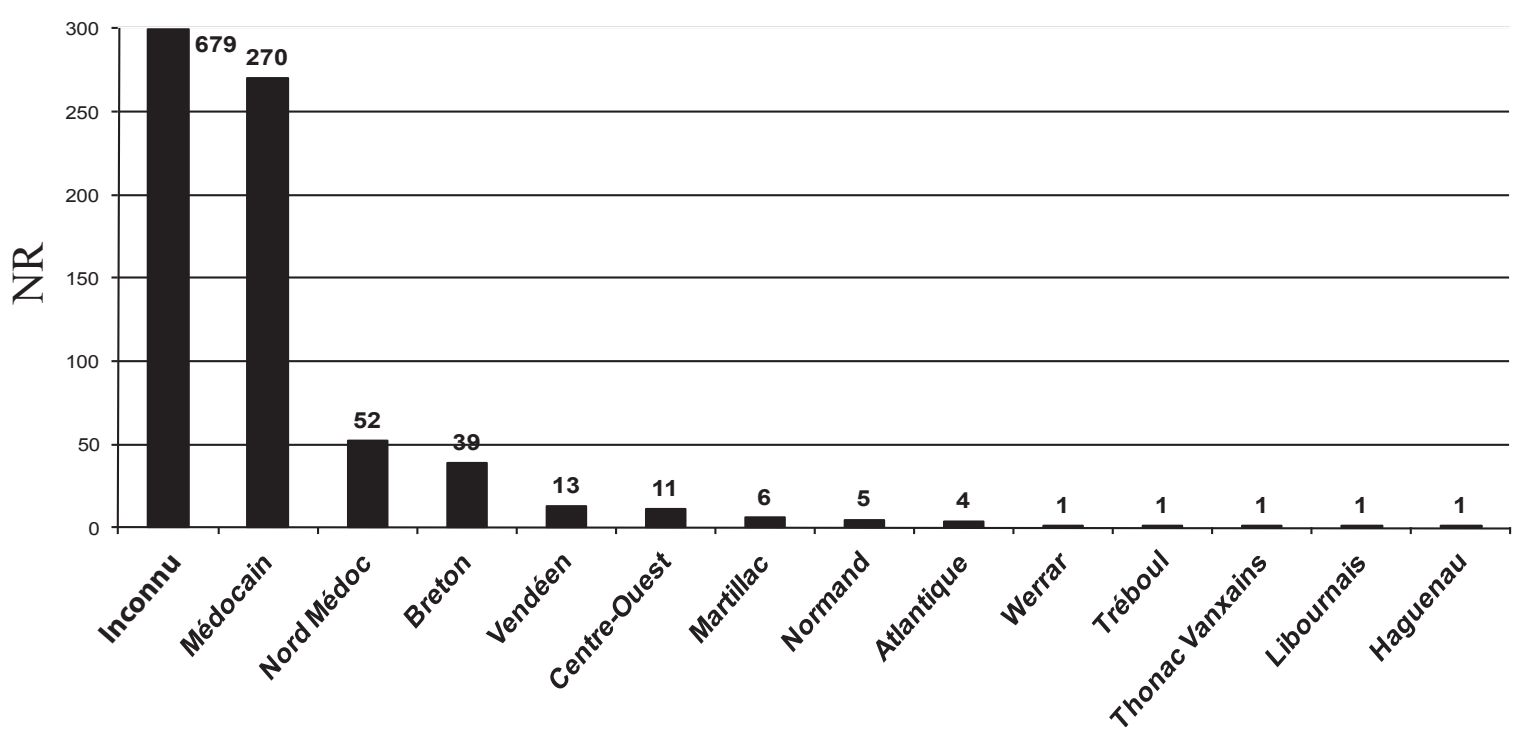

Fig. 14 - Diagrammes des types des haches rencontrés en Aquitaine et dans le Médoc (dépôts et découvertes isolées confondus). Fig. 14 - Diagram of axes from the Aquitaine and Médoc regions (hoards and isolated objects). 

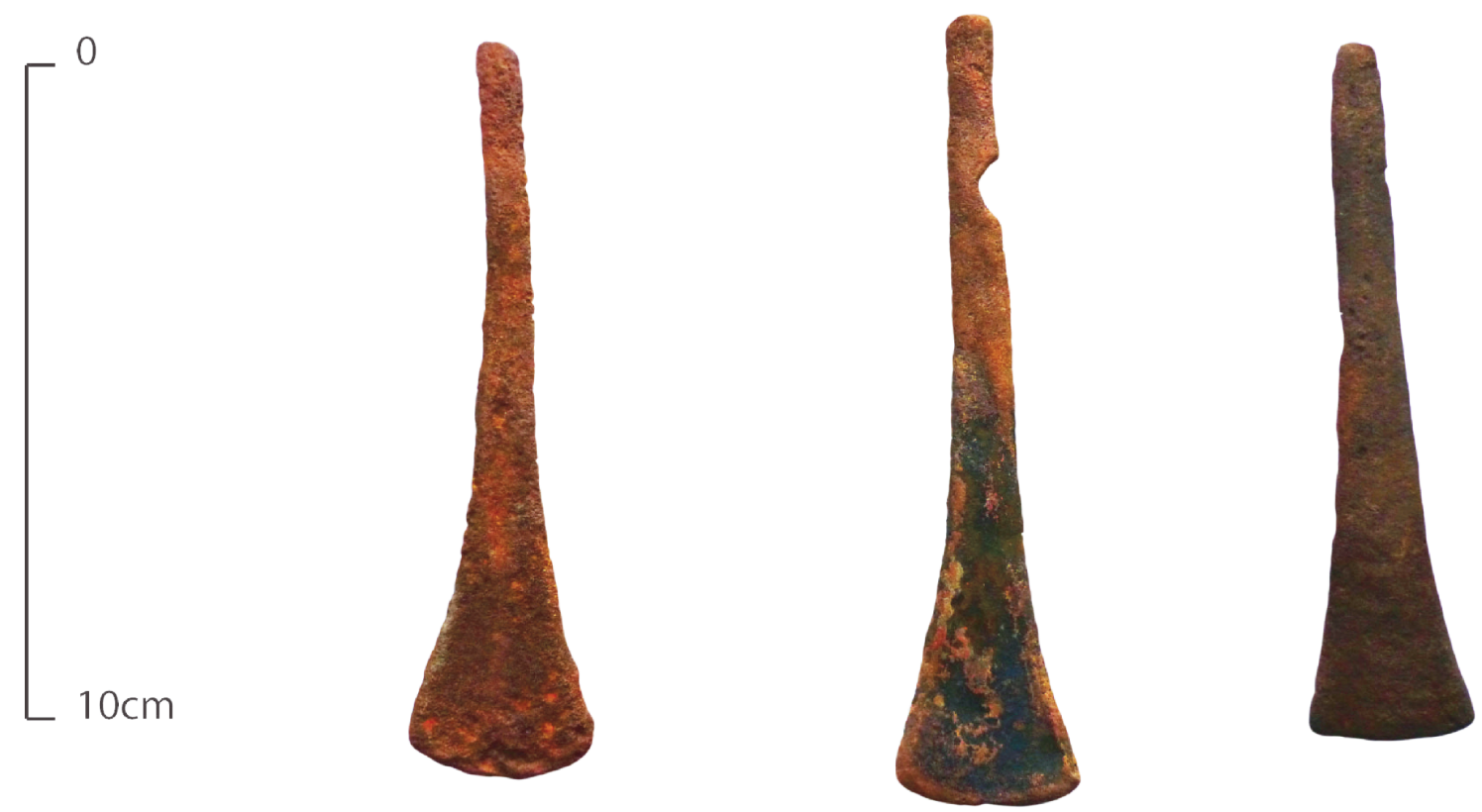

Fig.15 - Hachettes de Vendays-Montalivet du musée de la Grange du Patrimoine à Montalivet (clichés F. Couderc).

Fig. 15 - Small axes from Vendays-Montalivet in the Grange du Patrimoine museum, Montalivet (photos F. Couderc).

\section{LA PRATIQUE DES DÉPÔTS À LA LUMIÈRE DE L'ANALYSE SPATIALE}

$\mathrm{N}$ ous avons vu au début de cet article qu'il était possible à partir des données géomorphologiques et palynologiques de reconstituer une partie du paysage médocain de l'âge du Bronze. Durant cette période, l'environnement était beaucoup plus humide qu'aujourd'hui. L'étude des différentes cartes (géologiques, cadastrales) et des vues aériennes, couplées avec les données paléoenvironnementales, permet de redéfinir les contours des anciens marais de l'âge du Bronze moyen (fig. 16). Certains auteurs donnent quelques informations concernant le contexte de découverte des dépôts. Le dépôt de l'Aygua (Bégadan) a été découvert durant l'hiver 18931894. L'auteur précise que le dépôt a été découvert dans des terres de marais (Brion, 1896 et 1897). De plus, le terme d'aygua signifie localement « terres recouvertes d'eau ». Le dépôt du Chalet (Saint-Germain-d'Esteuil), a été découvert en 1999 dans un «bas-fond», que l'auteur désigne être comme un ancien marais (Roussot-Larroque, 1999). À l'inverse, certains dépôts ont été trouvés sur les terres hautes, comme les dépôts de Pauillac ou celui du Vignaud à Talais (Meynieu, 1881). Mais ces terres dominent les marais de quelques mètres seulement. Si ces dépôts n'étaient pas directement enfouis dans les marais ou sur leur bordure, ils n'étaient distants que de quelques dizaines de mètres de ces zones humides ou de l'estuaire (fig. 17). La localisation des objets métalliques isolés et en contexte inconnu du Bronze moyen au sens large se calque parfaitement à l'aire de répartition des dépôts (fig. 18). Bien qu'il soit difficile de savoir si ces objets ont été abandonnés intentionnellement, leur localisation à proximité des dépôts pourrait témoigner de l'existence de pratiques plus discrètes ou bien de dépôts plus massifs dont seuls quelques éléments erratiques ont été découverts. En revanche les découvertes effectuées dans des contextes domestiques et funéraires sont plus difficiles à interpréter, compte tenu de leur faible représentation en Médoc. Nous devons donc rester prudents sur ces découvertes anciennes dont les conditions de fouille sont inconnues.

Grâce aux systèmes d'information géographique, il est possible de redessiner les contours de ces marais de l'âge du Bronze et d'y replacer les différents dépôts dans leur contexte originel. Leur localisation reste assez approximative, généralement autour du lieu-dit. Il est difficile de considérer dans notre analyse les dépôts découverts sur la plage, car le trait de côte de l'âge du Bronze ne peut pas être situé précisément, et les données géomorphologiques des terres situées sous les dunes qui nous permettraient de restituer le paysage ancien ne sont pas disponibles. Seul le site de la Lède du Gurp (Grayan-et-l'Hôpital) situé sur la plage, semble se trouver en bordure d'un ancien chenal tidal recouvert par les dunes. Les dépôts de l'Amélie ont d'ailleurs été découverts à $200 \mathrm{~m}$ environ au nord de ce site.

Une analyse fine a été réalisée dans le cadre de cette étude autour de l'ancien marais de Saint-Laurent-deMédoc, situé sur la frange orientale du Médoc. Dans cette zone, plusieurs dépôts ont été découverts et leur localisation est relativement précise de 100 à $200 \mathrm{~m}$ près. Nous disposons aussi d'un relevé Lidar qui permet de mieux visualiser l'ancienne zone humide en connexion avec l'estuaire de la Gironde. Plusieurs données géomorphologiques issues de carottages sont disponibles dans ce secteur grâce à la base de données «InfoTerre » du BRGM. Il est possible de localiser les dépôts et les carottes sédimentaires dans un modèle en trois dimensions du marais 

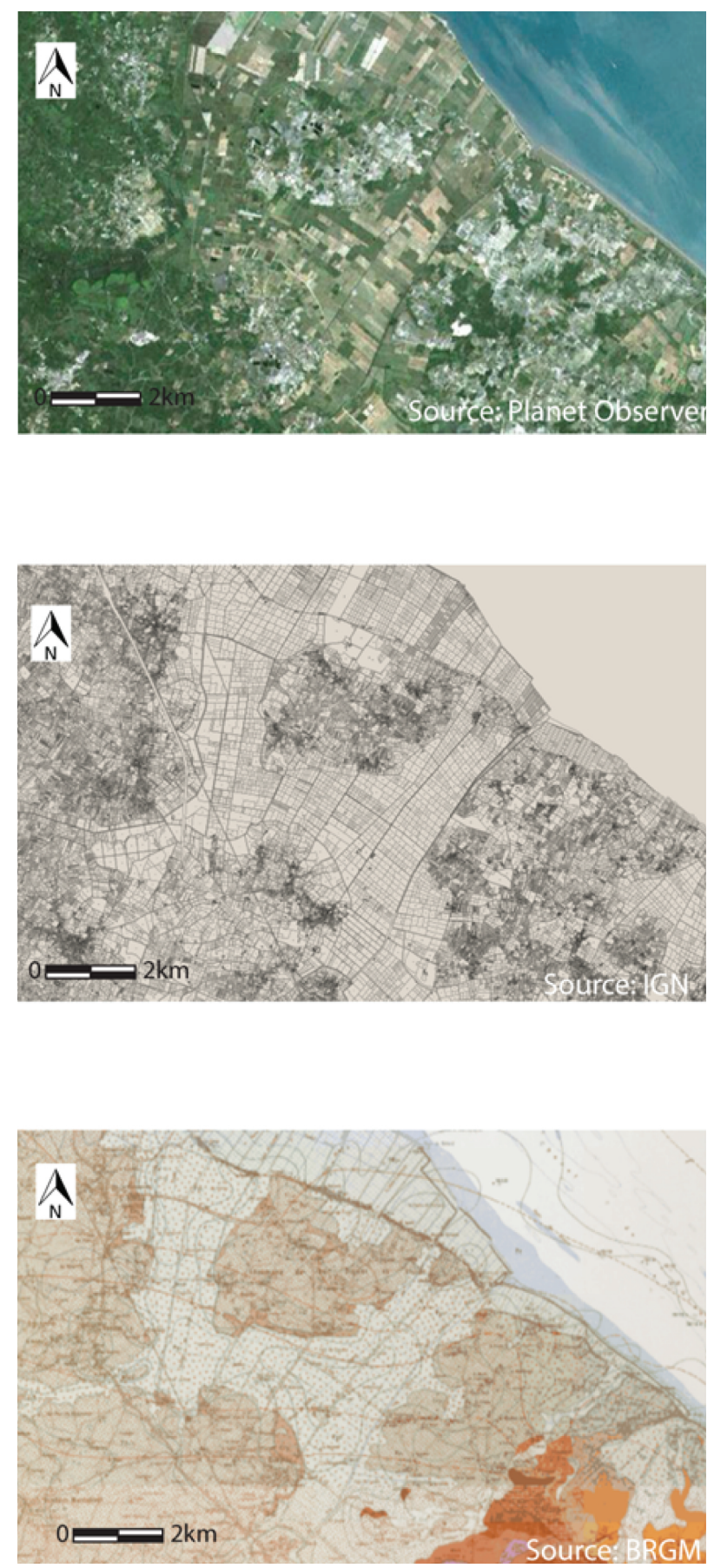

Fig. 16 - Vue aérienne, plan cadastral et carte géologique de Jau-Dignac-et-Loirac dans le nord-est du Médoc (IGN, BRGM).

Fig. 16-Aerial views, land register and geological maps of JauDignac-et-Loirac in the north-eastern Médoc (IGN, BRGM).

de Saint-Laurent, ce qui permet d'avoir une meilleure vision de l'ensemble de la zone d'étude. Le relief a légèrement été exagéré afin de marquer cette dépression naturelle, car l'altitude ne varie que de 0 à $30 \mathrm{~m}$ NGF. Nous ne disposons pas de données sur le couvert végétal des environs de ce marais, comme c'est le cas pour ceux du Nord du Médoc, mais ils devaient être approximativement identiques. Le marais de Saint-Laurent durant l'âge du Bronze devait ressembler aux rias bretonnes actuelles (fig. 19). Ces espaces topographiques ressemblent à de petites vallées en connexion avec l'océan ou un estuaire, dont les eaux subissent l'influence des marées.

Les données provenant des carottes du BRGM situées dans le marais illustrent la présence d'un sédiment très organique noir dans le premier mètre, et d'un comblement inférieur d'argiles, de sables puis de galets et gravillons (fig. 20 et 21). Les terrasses situées sur les bordures du marais sont, elles, exclusivement composées de graves, de sable ou d'argiles, puis de calcaire. Nous constatons que les dépôts sont uniquement situés en dehors du marais, dans des terres sèches, mais rarement éloignés de plus de quelques centaines de mètres de la zone humide (en prenant en compte la marge d'erreur de la localisation). Ils étaient donc toujours accessibles après leur enfouissement et n'étaient pas abandonnés aux eaux, comme cela peut être le cas dans d'autres contextes.

\section{DISCUSSION}

L es différents chercheurs ayant travaillé sur la question des dépôts métalliques en Médoc se sont le plus souvent concentrés sur les objets, sans réussir toutefois à proposer une interprétation de cette pratique si particulière de l'âge du Bronze. Grâce au nombre exceptionnel des découvertes réalisées depuis le XIX ${ }^{\mathrm{e}}$ siècle et à l'apport des données paléoenvironnementales, il est possible de restituer un comportement codifié dans la constitution et l'enfouissement des dépôts au Bronze moyen.

La localisation systématique des dépôts en bordure des zones humides n'est pas sans rappeler d'autres travaux réalisés sur la relation entre les sociétés protohistoriques et le milieu aquatique (Bradley, 1990; Fontijn, 2002; Ballmer, 2010; Mélin, 2011). Les recherches de Richard Bradley démontrent un lien direct entre l'abandon d'objets métalliques précieux et le milieu aquatique (rivières, lacs, tourbières). Il s'agirait d'une forme d'offrande aux divinités des eaux, élément qui semble avoir exercé très tôt un attrait particulier sur ces populations. Cette pratique est courante durant toute la Protohistoire et apparait dès le Chalcolithique, voire au Néolithique et se perpétue jusqu'aux périodes historiques et contemporaines. Ariane Ballmer développe l'idée d'une conception mentale du paysage des sociétés protohistoriques, en s'appuyant sur des exemples ethnographiques des sociétés saami et khanty du Nord-Est de l'Europe. Ces populations découpent leur territoire selon une codification qui leur est propre. Des espaces sont dédiés aux tâches du quotidien, tandis que d'autres sont plus liés au monde sacré et spirituel. Les dépôts réalisés par ces sociétés se placent généralement dans les espaces de transition entre ces deux mondes. Les dépôts peuvent également marquer des frontières entre des groupes humains ne partageant pas les même caractéristiques culturelles, comme cela a été proposé pour le Bronze final en France (Brun et al., 1997). Mais dans le cas du Médoc, l'estuaire de la Gironde joue déjà ce rôle, et les dépôts placés en bordure 


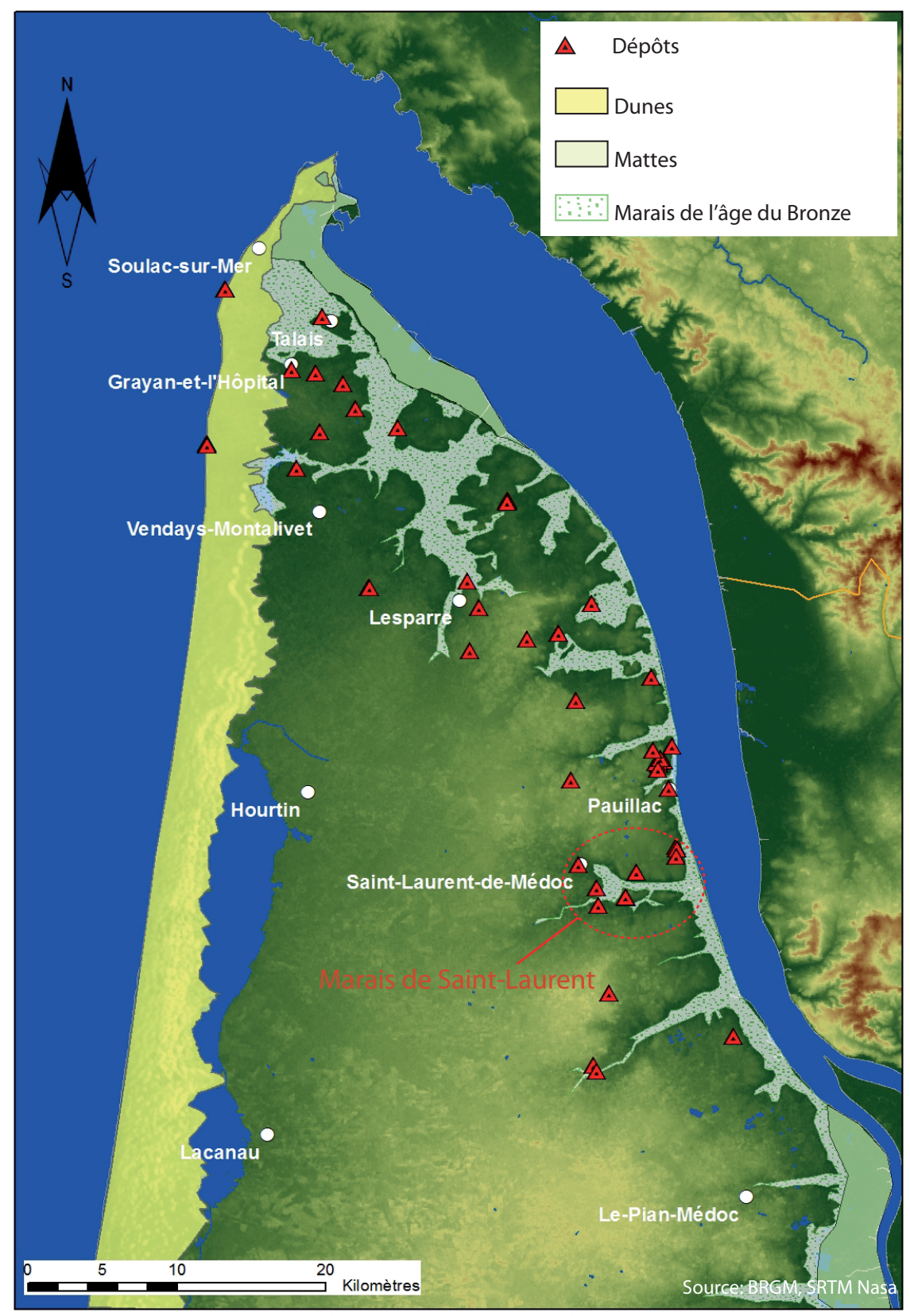

Fig. 17 - Localisation des dépôts du Bronze moyen 2 en Médoc, avec la restitution des marais de l'âge du Bronze moyen (SIG F. Couderc).

Fig. 17 - Location of Middle Bronze Age 2 hoards in Médoc, with the restitution of Middle Bronze Age marshes (GIS F. Couderc).

des zones humides marqueraient surtout une frontière entre le milieu aquatique et les terres émergées.

Les données sur l'habitat font défaut en Médoc, or leur localisation permettrait de comprendre l'organisation du territoire durant le Bronze moyen et de tenter de cerner la relation entre les dépôts et le paysage. Les recherches de David Fontijn mettent l'accent sur la relation entre l'objet, le paysage et les populations (Fontijn, 2002, p. 33-36). Il y voit un lien fort entretenu entre ces trois éléments et la pratique des dépôts durant l'âge du Bronze dans le Nord de l'Europe. La hache prend, comme pour le Médoc, une place importante aux Pays-Bas dans les dépôts du Bronze moyen. L'armement ne fait son apparition qu'à la fin de la période (Fontijn, 2002, p. 147). Il est intéressant de noter que dans les dépôts de haches des Pays-Bas, les productions locales sont privilégiées, mais que les importations européennes sont régulièrement présentes. En Médoc, le même rapport est constaté si l'on considère que les haches des types atlantiques et continentaux sont exogènes de par leur provenance ou bien par leur style emprunté à d'autres groupes culturels.

L'ouverture du Médoc aux échanges atlantiques est visible au travers des objets retrouvés dans les dépôts. Les haches des types breton, vendéen et normand regroupent respectivement trente-neuf, douze et cinq objets, alors que seules onze haches du type du Centre-Ouest et une hache du type d'Orléans ont été retrouvées. Les populations médocaines de l'âge du Bronze se sont surtout 


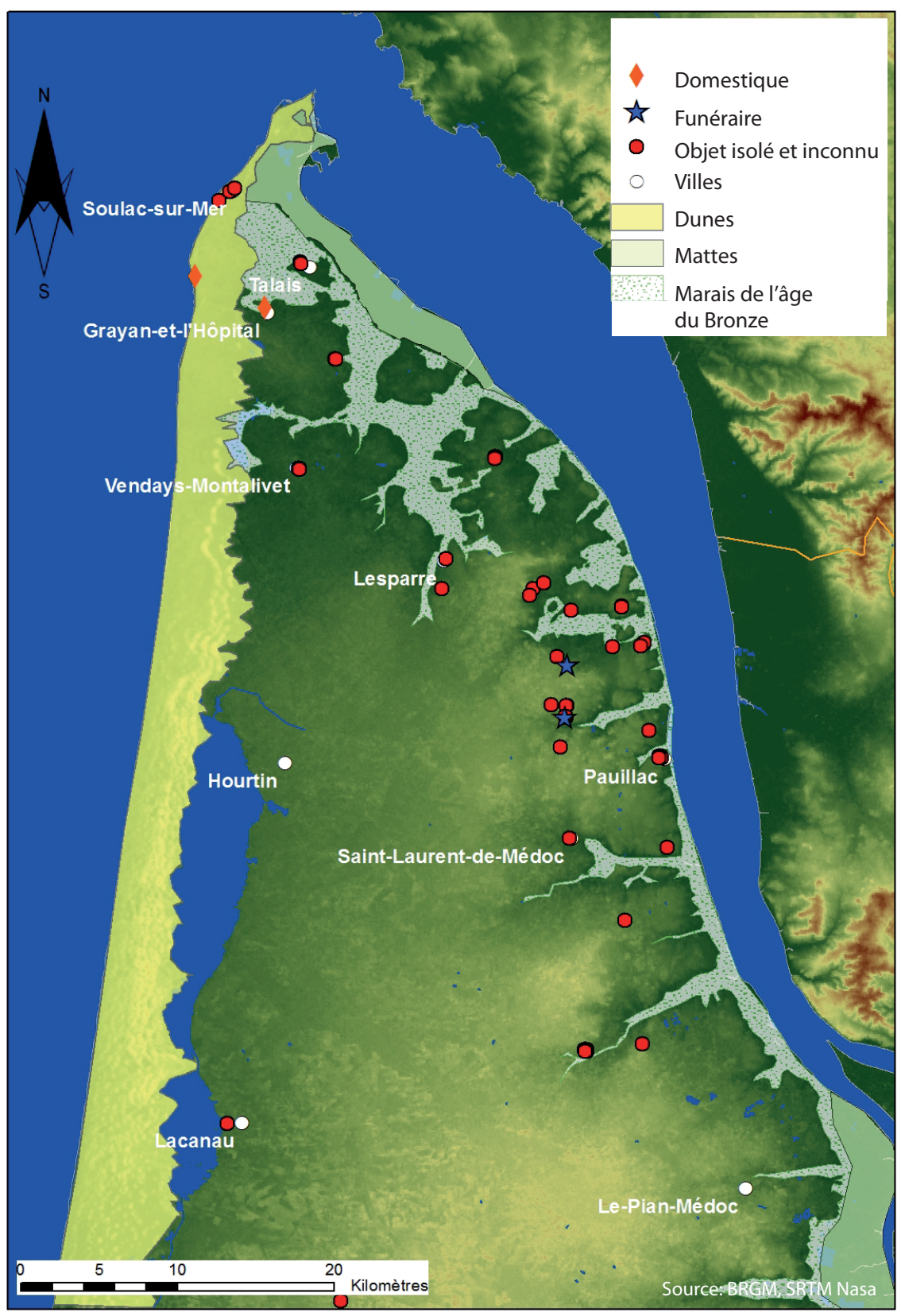

Fig. 18 - Localisation des objets isolés et des objets en contexte domestique et funéraire du Bronze moyen en Médoc, avec la restitution des marais de l'âge du Bronze moyen (SIG F. Couderc).

Fig. 18 - Location of Middle Bronze Age isolated objects, and objects from domestic and funerary contexts in Médoc, with the restitution of Middle Bronze Age marshes (GIS F. Couderc).

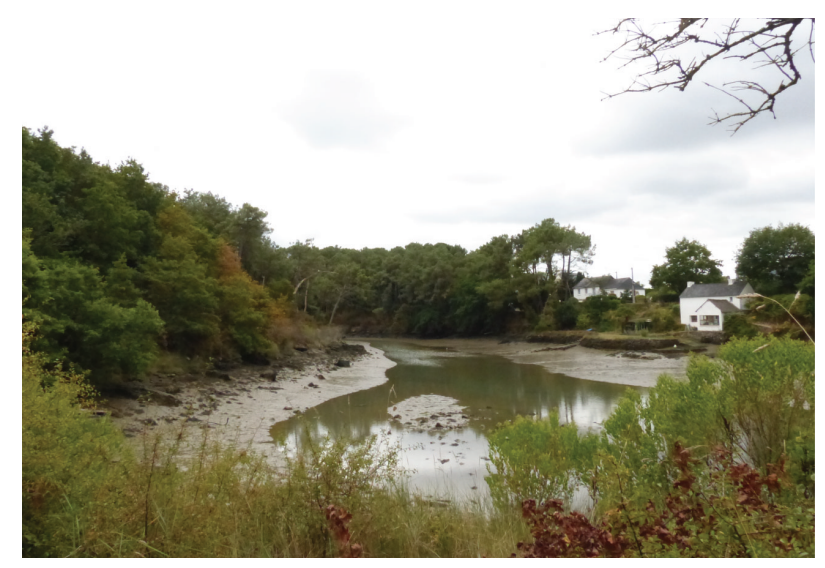

tournées vers l'océan, qui offrait certainement de meilleures opportunités pour les échanges que le centre de la France. L'attractivité du Médoc s'explique certainement par son emplacement stratégique aux portes du golfe de Gascogne, réputé pour être difficile à naviguer, et par sa localisation en aval de la Garonne qui constitue un axe de circulation privilégié pour rejoindre les terres intérieures

Fig. 19 - Exemple d'une ria bretonne en Morbihan à Moëlansur-Mer (cliché F. Couderc).

Fig. 19 - Example of a Breton ria in Morbihan, Moëlan-surMer (photo F. Couderc). 


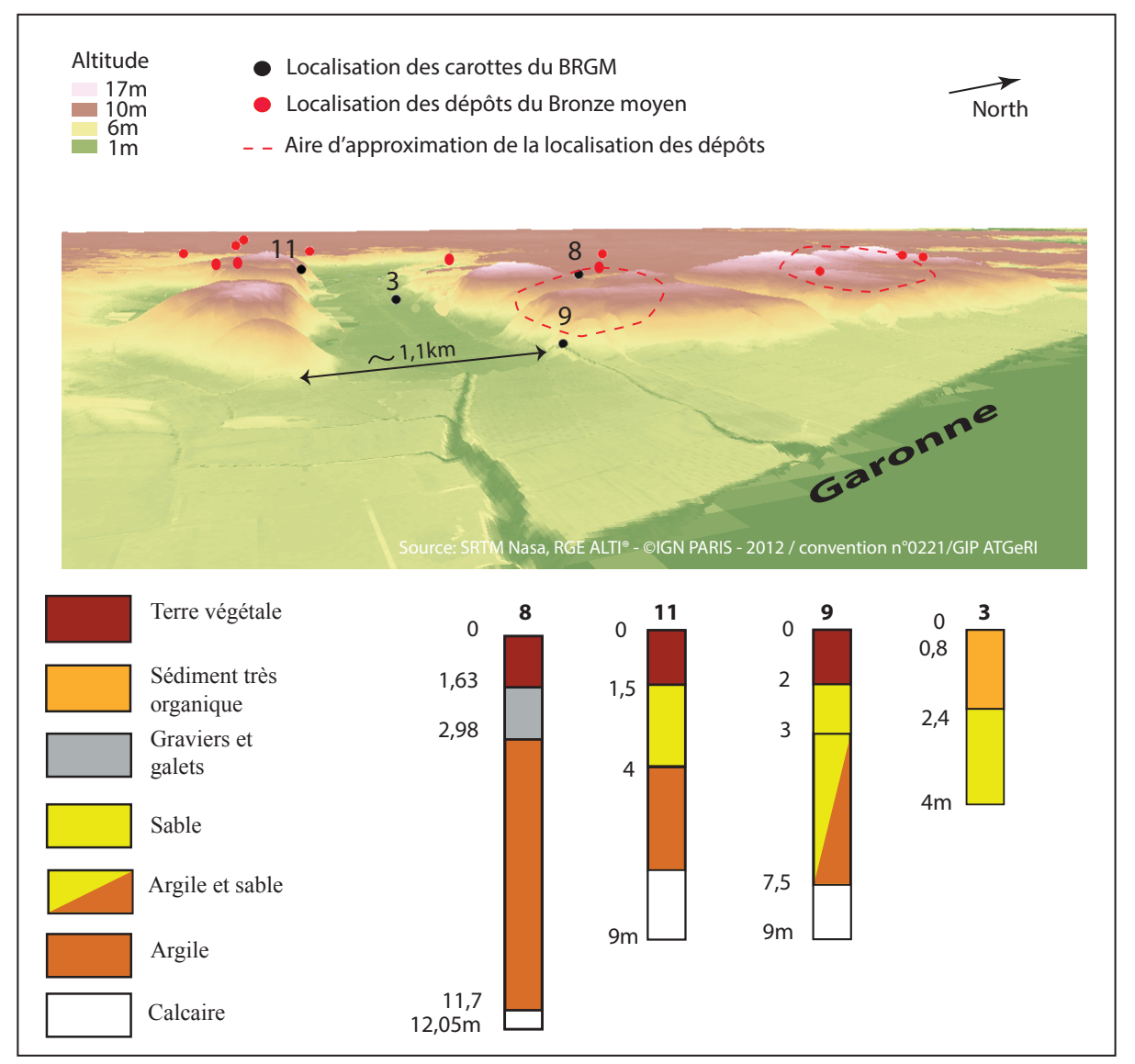

Fig. 20 - Restitution 3D du marais de Saint-Laurent-de-Médoc avec la localisation des dépôts et des carottes du BRGM, vue depuis le sud-ouest (SIG F. Couderc).

Fig. 20 :3D restitution of Saint-Laurent-de-Médoc marshland with the localisation of hoards and core samples, view from the southwest (GIS F. Couderc).

du Sud-Ouest de la France. Le Médoc pouvait ainsi importer la matière première nécessaire à la confection des objets métalliques, et participer aux échanges avec le reste des sociétés du Bronze moyen atlantique. Un moule de hache à rebords, un moule d'enclume bigorne en gneiss du type de Porcieu-Amblagnieu sur le site de la Lède du Gurp (fig. 22) ainsi que des déchets de métallurgie témoignent d'une production d'objets en bronze en Médoc. La richesse des échanges pourrait se matérialiser ici par une importante pratique des dépôts. Cependant ces objets enfouis peuvent aussi être considérés comme rares et précieux ce qui augmente la valeur symbolique des dépôts.

Leur localisation à proximité des marais, témoigne d'une pratique ritualisée, car au travers de tout le territoire les dépôts seront systématiquement composés des mêmes objets et placés dans les mêmes espaces parfois distants de seulement quelques mètres. Cette constante n'est pas propre au Médoc ou au Bronze moyen (Tabbagh et Verron, 1983; Gabillot et Gomez de Soto, 2003 et 2004), car on la retrouve entre autre en Bretagne où durant le Bronze final la composition des dépôts semblent répondre à des standards de composition et de localisation. Mais dans le cas du Médoc au Bronze moyen, cette standardisation de la pratique des dépôts est corrélée par les données paléoenvironnementales. Ce choix dans l'emplacement des dépôts illustre certainement un lien entre les sociétés du Bronze moyen et les marais estuariens et côtiers. Si ce constat est évident au travers de l'analyse spatiale, l'essence même de cette pratique reste néanmoins difficile à appréhender pour notre mode de pensée contemporain.

Il reste un point crucial à aborder. La question de la contrepartie que pouvaient proposer les populations du Médoc en échange des ressources métalliques. Nous ne connaissons malheureusement que de très rares indices de sites d'habitat ou funéraire, qui nous permettraient de cerner les modalités d'occupation du sol et le système économique de ces populations. Seuls quelques indices d'habitat et de pratiques agricoles nous sont parvenus sur le site de la Lède du Gurp, où des traces d'araire apparaissent dans les sols fossilisés sous les dunes côtières (Roussot-Larroque, 2007).

À la dune du Pilat, près du bassin d'Arcachon et dans le Nord du Médoc sur la plage de l'Amélie, des fragments de briquetage, des foyers et des traces de rubéfactions indiqueraient une production de sel dès le Bronze moyen, mais surtout durant l'âge du Fer (Verdin et al., 2015, p. 883). Le sel marin pourrait être le socle économique sur lequel les sociétés de l'âge du Bronze se seraient construites. 


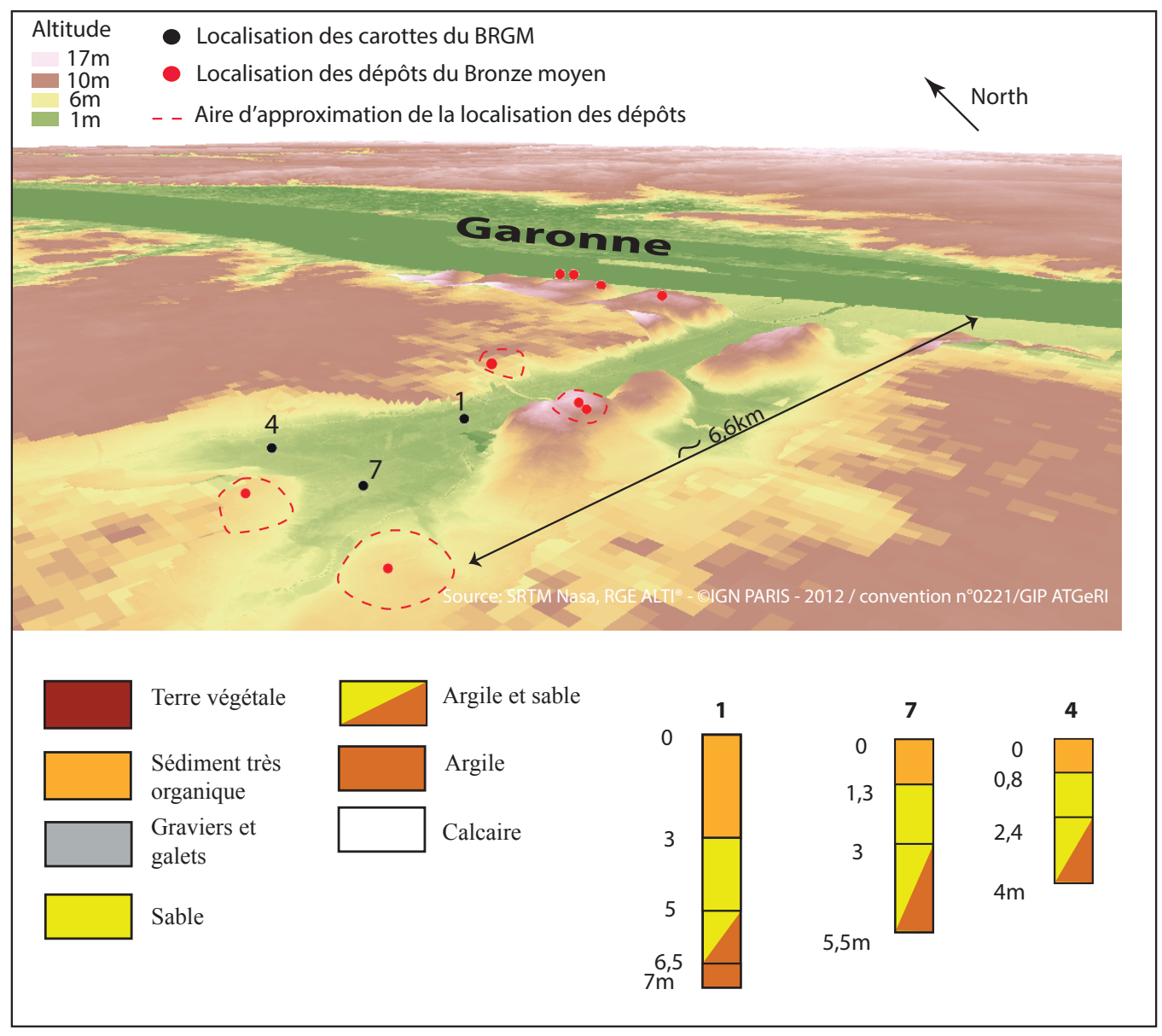

Fig. 21 - Restitution 3D du marais de Saint-Laurent-de-Médoc avec la localisation des dépôts et des carottes du BRGM, vue depuis l'est (SIG F. Couderc).

Fig. 21 - 3D restitution of Saint-Laurent-de-Médoc marshland with the localisation of hoards and core samples, view from the east (GIS F. Couderc).

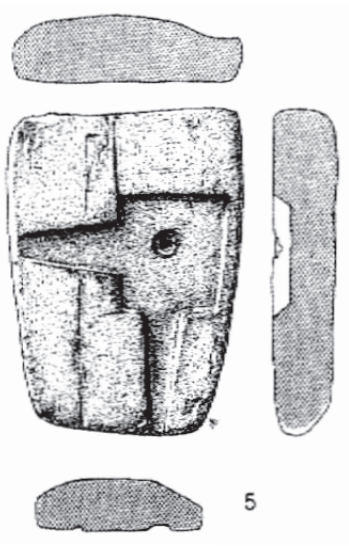

1
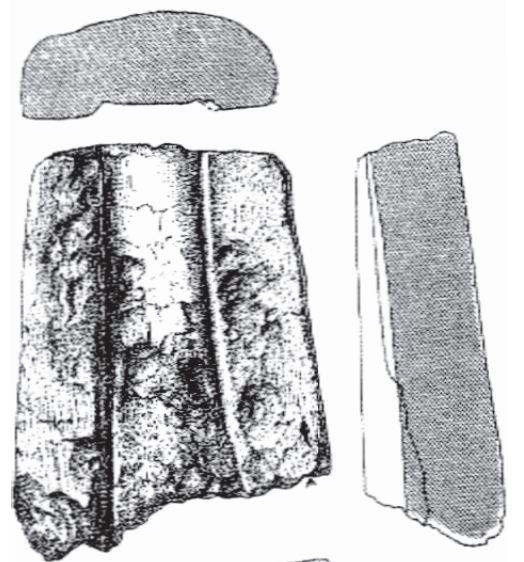

$5 \mathrm{~cm}$

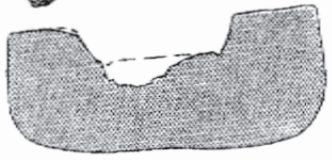

2

Fig. 22 - 1 : moule d'enclume bigorne de la plage de la Balise à Soulac-sur-Mer; 2: moule de hache à rebords de la Lède du Gurp (d'après Roussot-Larroque, 1997).

Fig. 22 - 1: Mould of an anvil from Balise beach, Soulac-sur-Mer; 2: mould of a flanged axe from Lède du Gurp (after RoussotLarroque, 1997). 
Nous savons qu'il a joué et joue encore pour de nombreuses populations à travers le monde le rôle de monnaie d'échange car le sel est utilisé pour l'alimentation des bêtes et pour la conservation de certains aliments. Cette hypothèse pourrait expliquer la baisse significative des dépôts au Bronze final en Médoc. Car, comme nous l'avons montré dans cet article, c'est durant cette période que l'environnement va devenir de plus en plus humide, avec des tempêtes à répétition qui formeront le cordon de Richard. La modification du paysage a pu influencer les sociétés protohistoriques et perturber leur fonctionnement économique au Bronze final. C'est en effet durant cette période que les dépôts vont pratiquement disparaître en Médoc, alors que sur l'ensemble de la façade atlantique, le Bronze final voit une recrudescence des dépôts métalliques, principalement durant le Bronze final 3 (Roussot-Larroque, 2001 et 2003 ; Mélin, 2011; Milcent, 2012). Les quelques dépôts connus de cette période en Aquitaine (majoritairement pour le Bronze final 2) se retrouvent plutôt en amont de l'estuaire, à l'intérieur des terres. Les réseaux d'échange ont certainement été modifiés durant le Bronze final, ne faisant plus du Médoc une zone économique majeure. Mais une absence de dépôts au Bronze moyen 1 et au Bronze final n'est pas forcément synonyme d'une faible dynamique ou d'un essoufflement des échanges. $\mathrm{Au}$ contraire une rareté des produits métalliques peut amener les sociétés protohistoriques à prêter aux objets une forte valeur symbolique ou économique et les poussent à constituer des dépôts. Cependant à l'échelle de la façade atlantique la pratique des dépôts est exponentielle du Bronze moyen jusqu'au Bronze final, alors qu'en Médoc un arrêt brutal est perceptible. Il est difficilement concevable pour des sociétés traditionnelles qu'un tel changement des pratiques ne soit pas associé à une baisse des activités économiques et à un effondrement des élites sociales du Bronze moyen, qui vraisemblablement contrôlaient les échanges et la redistribution des biens métalliques. La question reste cependant ouverte sur l'origine de cette pratique au début du Bronze moyen, car durant le Bronze ancien en Médoc la pratique des dépôts métalliques semble inexistante ou du moins imperceptible.

\section{CONCLUSION}

T a fonction symbolique des dépôts est toujours difficile à aborder, car le sens même d'une pratique ritualisée dans des sociétés traditionnelles, où le quotidien et le religieux se mêlent constamment, nous est difficilement accessible. Cependant, dans le cas du Médoc, le lien entre le paysage et cette pratique est bien attesté. La multiplication des dépôts est probablement à mettre en relation avec la place stratégique du Médoc dans les circuits d'échanges du Bronze moyen sur la façade atlantique. Les populations ont pu profiter de la ressource naturelle abondante qu'était le sel. Mais sa production a pu être perturbée par un important changement de l'environnement au Bronze final, empêchant les populations de disposer des ressources nécessaires à un maintien aussi intense des contacts avec le reste du monde atlantique, et perdant ainsi largement la possibilité de s'approvisionner en produits métalliques.

Bien que le cas du Médoc soit particulier à l'échelle de la France par la surabondance des dépôts, les comparaisons avec d'autres régions de France et d'Europe permettent d'entrevoir des comportements similaires pendant le Bronze moyen. Ces dépôts témoignent de l'importance des réseaux d'échange sur la façade atlantique et de leur difficulté à s'adapter face aux contraintes environnementales. Mais durant le Bronze final, un important changement est perceptible au travers de l'absence de dépôts de cette période en Médoc, alors que dans le même temps ils se multiplient sur l'ensemble de la façade atlantique.

Le cas du Médoc à bien des égards ne peut être transposé au reste de l'Europe occidentale, mais il permet toutefois d'ouvrir de nouvelles pistes de réflexion qui devront être étayées par de nouvelles recherches sur le terrain et en laboratoire.

Remerciements : Cette recherche n'aurait jamais pu aboutir sans l'appui d'Alexis Gorgues, de Pierre-Yves Milcent, de Francis Bordas et de Fabrice Marembert. Je tiens à les remercier ici, ainsi que Céline Lagarde-Cardona pour ses recherches qui représentent un socle important sur lequel s'appuie cette étude. Je remercie également Lionel Rubele et Anne-Sophie Murray pour leurs corrections de l'anglais.

\section{RÉFÉRENCES BIBLIOGRAPHIQUES}

Allen J.-P., Castaing P., Feral A., Klingebiel A., VigneauX M. (1970) - Contribution à l'étude des faciès de comblement et interprétation paléogéographique de l'évolution des milieux sédimentaires récents et actuels de l'estuaire de la Gironde, Bulletin de l'Institut de géologie du bassin d'Aquitaine, 8, p. 99-154.

Ballmer A. (2010) - Measuring the Mental: A Quantitative Approach to Mental Landscape Concepts, in Human development in landscapes, Landscapes and Human Development: The Contribution of European Archaeology, actes de l'atelier interntional « Socio-Environmental Dynamics over the Last 12,000 Years: The Creation of Landscapes » (Kiel,

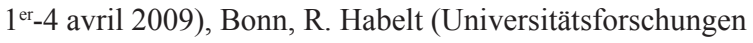
zur prähistorischen Archäologie, 191), p. 193-202.

Berchon E. (1890) - Études paléo-archéologiques sur l'âge du Bronze, spécialement en Gironde, Bordeaux, Cadoret, $245 \mathrm{p}$.

Bradley R. (1990) - The Passage of Arms: An Archaeological Analysis of Prehistoric Hoards and Votive Deposits, Cambridge, Cambridge University Press, 234 p. 
Brion A. (1896) - Notice sur une cachette de l'époque du Bronze découverte à Bégadan, Bulletin et mémoires de la Société archéologique de Bordeaux, 21, p. 303-305.

BRION A. (1897) - Note supplémentaire sur une cachette de l'époque du Bronze découverte à Bégadan, Bulletin et mémoires de la Société archéologique de Bordeaux, 22, p. 115-116.

Brun P., Aubry F., Giraud F., Lepage S. (1997) - Dépôts et frontières au Bronze final en France, Boletin del Seminario de Estudios de Arte y Arquelogia, 63, p. 97-114.

CAIla P. de (1806) - Dissertation sur un instrument antique trouvé dans la paroisse de Pauillac-en-Médoc, Gironde, en mars 1803, Actes de l'académie de Bordeaux, 3, p. 167-208.

Charrol M. (1912) - Haches en bronze de Saint-Laurent (Médoc), Bulletin et mémoires de la Société archéologique de Bordeaux, 37, p. 146-152.

Clavé B. (2001) - Évolution des paléo-environnements côtiers à l'Holocène : exemple de l'Aquitaine septentrionale, thèse de doctorat, université Bordeaux I, $310 \mathrm{p}$.

CofFyn A. (1972) - Le Bronze final et le début du premier âge du Fer autour de l'estuaire Girondin, thèse de doctorat, université Bordeaux III, 499 p.

Coffyn A. (1989) - En Médoc, avant le vin, le Bronze, Fédération historique du Sud-Ouest, 16, p. 45-57.

Coffyn A., Gachina J. (1972) - Un dépôt de l'âge du Bronze à Saint-Vivien-de-Médoc (Gironde), Bulletin de la Société préhistorique française, 74, p. 269-278.

Coffyn A., Moreau J., Bourhis J.-R. (1993) - Quelques bronzes girondins inédits ou peu connus, Revue archéologique de Bordeaux, 34, p. 57-78.

Coffyn A., Moreau J., Bourhis J.-R. (1995) - Les dépôts de bronze de Soulac-sur-Mer (Gironde), Aquitania, 13, p. 7-31.

Coquillas D. (2001) - Les rivages de l'estuaire de la Gironde, du néolithique au Moyen Âge, thèse de doctorat, université Bordeaux III, $1296 \mathrm{p}$.

Coquillas D., Diot M.-F., Clave B., Tastet J.-P. (2006) - L'homme dans les paysages côtiers du Nord-Médoc (Gironde), entre Néolithique et âge du Fer, in P. Fouéré, C. Chevillot et P. Courtaud (éd.), Paysages et peuplements : aspects culturels et chronologique en France méridionale, actualités de la recherche, actes des $\mathrm{VI}^{\mathrm{es}}$ Rencontres méridionales de Préhistoire récente (Périgueux, juin 2004), Périgueux, ADRAHP (Préhistoire du Sud-Ouest, supplément $11)$, p. 55-70.

Daleau F. (1876) - Carte archéologique du département de la Gironde, Association française pour l'avancement des sciences, 5, p. 607-618.

Daleau F. (1896) - Cachette de l'âge du Bronze découverte au Barrail, commune de Braud, Bulletin et mémoires de la Société archéologique de Bordeaux, 21, p. 7-13.

Devignes M., Coffyn A. (1987) - Haches médocaines de Lacanau (Gironde), Société archéologique de Bordeaux, 28, p. 25-28.

DiOT M.-F. (1991) - Apport de la palynologie à la connaissance de l'environnement de l'âge du Bronze dans le Sud-Ouest de la France, in C. Chevillot et A. Coffyn (dir), L'âge du
Bronze atlantique. Ses faciès, de l'Écosse à l'Andalousie et leurs relations avec le Bronze continental et la Méditerranée, actes du I ${ }^{\text {er }}$ Colloque du parc archéologique de Beynac (Beynac-et-Cazenac, 10-14 septembre 1990), Beynacet-Cazenac, AMUSA, p. 319-332.

Diot M.-F, TAstet J.-P. (1995) - Paléoenvironnements holocènes et limites chronoclimatiques enregistrées dans un marais estuarien de la Gironde (France), Quaternaire, 6, 2, p. 85-94.

Diot M.-F., Masse L., Coquillas D., Clave B. (2002) - Utilisation des groupes polliniques dans la reconstitution des paléoenvironnements holocènes du marais de la Perge-Nord Médoc-Gironde (France). Implication sur l'occupation humaine, Revue d'archéométrie, 26, 1, p. 155-169.

FILy M. (2008) - Les monuments funéraires et les dépôts métalliques dans le paysage rituel de l'âge du Bronze : l'exemple $d u$ Centre-Ouest de la Bretagne et du Finistère littoral (France), thèse de doctorat, université Rennes 1, 621 p.

Fontisn D. (2002) - Sacrificial Landscapes. Cultural Biographies, Objects and 'Natural' Places in the Bronze Age of the Southern Netherland, c. 2300-600 BC, Leyde, University of Leiden (Analecta Praehistorica Leidensia, 33-34), 392 p.

Frugier G., Andrieux P., Boudet R. (1983) - Les moules à bronze de l'habitat de la Lède du Gurp (Gironde), in Journées de paléométallurgie, actes du colloque (Compiègne, 22-23 février 1983), Compiègne, Université de technologie, p. $447-465$.

Gabillot M. (2003) - Dépôts et production métallique du Bronze moyen en France nord-occidentale, Oxford, Archaeopress, $471 \mathrm{p}$.

Gabillot M., Gomez de Soto J. (2003) - Dépôts de l'âge du Bronze et du premier âge du Fer en Gaule de l'Ouest, de la Manche à l'Aquitaine septentrionale. Découvertes et recherches récentes, nouvelles perspectives, Documents d'archéologie méridionale, 26, p. 357-364.

Gabillot M., Gomez de Soto J. (2007) - Trésors et cachettes de l'âge du Bronze en France : cent ans de recherches et d'évolution des méthodes d'analyse, in J. Évin (dir.), Un siècle de construction du discours scientifique en Préhistoire, actes $\mathrm{du} \mathrm{XXVI}^{\mathrm{e}}$ Congrès préhistorique de France, congrè du centenaire de la Société préhistorique française (Avignon, 21-25 septembre 2004), Paris, SPF, vol. 2, p. 55-69.

Gabillot M., Monna F., Alibert P., Bohard B., Camizuli E., Dommergues C.-H., Dumontet A., Forel B., Gerber S., Jebrane A., Laffont R., Navarro N., Specht M., Chateau C. (2017) - Productions en série vers 1500 avant notre ère. Des règles de fabrication au Bronze moyen entre la Manche et les Alpes à la lumière d'une étude morphométrique, in. C. Mordant et S. Wirth (dir.), Normes et variabilités au sein de la culture matérielle de l'âge du Bronze, actes de la journée thématique de la Société préhistorique française organisée avec l'Association pour la promotion des recherches sur l'âge du Bronze (Dijon, 15 juin 2013), Paris, SPF (Séances de la Société préhistorique française, 10), p. 19-31.

Galinand C. (2008) - Usage social et symbolique du métal en France au Bronze ancien et moyen (2200-1350 av. J.-C.), 
thèse de doctorat, université Paris 1 - Panthéon-Sorbonne, $507 \mathrm{p}$.

Gomez de Soto J. (1980) - Les Cultures de l'âge du Bronze dans le bassin de la Charente, Périgueux, Fanlac, 120 p.

Gomez de Soto J. (1995) - Le Bronze moyen en Occident. La culture des Duffaits et la civilisation des Tumulus, Paris, Picard (L'âge du Bronze en France, 5), 375 p.

JoUANNET F.-V. (1827-1828) - Rapport sur quelques instruments en bronze trouvés à Pauillac en 1803 et en 1822, Actes de l'académie de Bordeaux, 8, p. 24.

Lagarde-Cardona C. (2012) - Production métallique en Aquitaine à l'âge du Bronze moyen. Techniques, usages et circulation, Pessac, Ausonius (Scipta Antiqua, 39), 420 p.

LALANNe G. (1887) - L'homme préhistorique dans le bas Médoc, Bulletin de la Société d'anthropologie de Bordeaux, 3, p. 97-141.

Melin M. (2011) - Les dépôts d'objets métalliques en milieu humide pendant l'âge du Bronze en France. Caractérisation des pratiques d'immersion, thèse de doctorat, université Rennes 1, 475 p.

Melin M. (2014) - Le dépôt du Bronze moyen II de Mouilleron-en-Pareds (Vendée) et les haches à rebords du type vendéen, Revue archéologique de l'Ouest, 31, p. 83-106.

Meynieu A. (1881) - Nouvelle cachette de l'âge du Bronze à Talais, Matériaux pour l'histoire primitive et naturelle de l'homme, 2, p. 491.

Milcent P.-Y. (2012) - Le temps des élites en Gaule atlantique : chronologie des mobiliers et rythmes de constitution des dépôts métalliques dans le contexte européen, $X I I I^{e}-V I I^{e}$ siècle av. J.-C., Rennes, Presses universitaires de Rennes, $253 \mathrm{p}$.

Mortillet G. (1894) - Cachettes de l'âge du Bronze en France, Bulletin de la Société d'anthropologie de Paris, 5, 1, p. 298-340.

Pautreau J.-P. (1979) - Le Chalcolithique et l'âge du Bronze en Poitou (Vendée, Deux-Sèvres, Vienne), Poitiers, Centre d'archéologie et d'ethnologie poitevines, musée SainteCroix, $431 \mathrm{p}$.

Pontee N.-I., Tastet J.-P., Masse L. (1998) - Morpho-Sedimentary Evidence of Holocene Coastal Changes near the Mouth of the Gironde and on the Médoc Peninsula, SW France, Oceanologica Acta, 21, 2, p. 243-261.

Roussot-Larroque J. (1982) - Pour un musée préhistorique imaginaire, Bulletin de la Société archéologique de Bordeaux, 73, p. 21-49.

Roussot-LARroque J. (1988) - Fouille pré- et protohistorique à la Lède du Gurp (Grayan-et-l'Hôpital, Gironde), Revue archéologique de Bordeaux, 79, p. 19-60.

Roussot-Larroque J. (1989) - Le dépôt du Bronze moyen de la Clare ou l'Aygua à Bégadan (Gironde), L'Anthropologie, 93, 1, p. 261-278.

Roussot-Larroque J. (1997) - La Lède du Gurp et la métallurgie du Bronze moyen en Médoc (annexes par J. Bourhis), Revue archéologique de Bordeaux, 88, p. 33-56.
Roussot-Larroque J. (1999) - Saint-Germain-d'Esteuil, le Grand Bois, Bilan scientifique Aquitaine, 9, p. 55-56.

Roussot-Larroque J. (2001) - Bronze moyen et final autour de l'estuaire de la Gironde, in J. Briard et J. L'Helgouach (éd.), Systèmes fluviaux, estuaires et implantations humaines, de la Préhistoire aux grandes invasions, actes du $124^{\text {e }}$ Congrès national des sociétés historiques et scientifiques (Nantes, 1999), Paris, CTHS, p. 253-271.

Roussot-Larroque J. (2003) - Le Bronze Atlantique entre Loire et Pyrénées : données et problèmes, in R. Desbrosse et A. Thevenin (éd.), Préhistoire de l'Europe. Des origines à l'âge du Bronze, actes du $125^{\mathrm{e}}$ Congrès national des sociétés savantes et scientifiques (Lille, 2000), Paris, CTHS, p. 535566 .

Roussot-Larroque J. (2007) - Le temps qui passe et le temps qu'il fait : prises et déprises agricoles en Médoc durant l'âge du Bronze, in H. Richard, M. Magny et C. Mordant (éd.), Environnements et cultures à l'âge du Bronze en Europe occidentale, actes du $129^{\mathrm{e}}$ Congrès national des sociétés savantes historiques et scientifiques (Besançon, 2004), Paris, CTHS, p. 285-293.

Roussot-Larroque J., Bourhis J.-R, Briard J. (2001) - Une production originale de l'âge du Cuivre dans le Médoc : pointes de Palmela et hachettes minces de VendaysMontalivet (Gironde) in J. Briard et J. L'Helgouach (éd.), Systèmes fluviaux, estuaires et implantations humaines, de la Préhistoire aux grandes invasions, actes du $124^{\mathrm{e}}$ Congrès national des sociétés historiques et scientifiques (Nantes 1999), Paris, CTHS, p. 273-284.

Sion H. (1989) - François Daleau et l'âge du Bronze en Médoc, Fédération historique du Sud-Ouest, 16, p. 59-75.

Tabbagh A., Verron G. (1983) - Étude par prospection géomagnétique de trois sites à dépôts de l'âge du Bronze, Bulletin de la Société préhistorique française, 80, 10-12, p. 375389.

Tastet J.-P., Roussot-Larroque J., Clave B. (2000) - Palaeoenvironmental Study Area P15: West Bank of the Gironde Estuary, West Coast, France, in R. G. McInnes, D. J. Tomalin et J. Jakeways (éd.), LIFE Environment Project: LIFE 97 ENV/UK/000510 1997-2000 Coastal Change, Climate and Instability: Final Technical Report, Newport, Isle of Wight Center for the Coastal Environment, 57p.

Verdin F., Dumas A., Hiriart E. (2015) - Entre terre et eau : le Médoc à l'âge du Fer, in F. Olmer et R. Roure (éd.), Les Gaulois au fil de l'eau, actes du XXXVIII Colloque de l'Association française de l'étude de l'âge du Fer (Montpellier, 8-11 mai 2013), Pessac, Ausonius, p. 881-892.

Florian COUdERC doctorant UMR 5608 TRACES, université Toulouse 2 - Jean-Jaurès, équipe RHAdAMANTE florian.couderc@hotmail.fr 